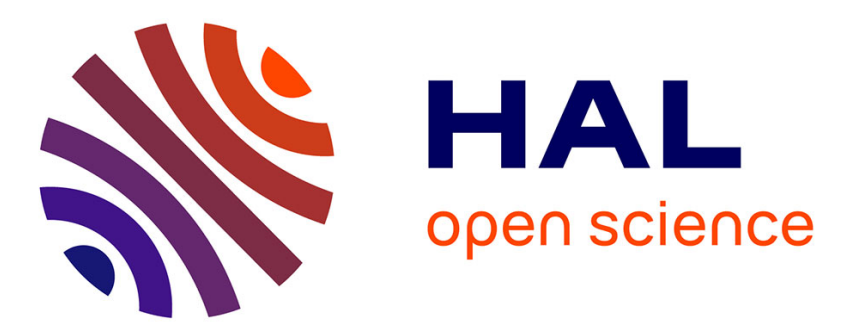

\title{
Electronic and physico-chemical properties of nanometric boron delta-doped diamond structures
}

Gauthier Chicot, Alexandre Fiori, P.N. Volpe, Thu Nhi Tran Thi, Jean-Claude Gerbedoen, Jessica Bousquet, M.P. Alegre, J.C. Pinero, D. Araujo, F. Jomard, et al.

\section{To cite this version:}

Gauthier Chicot, Alexandre Fiori, P.N. Volpe, Thu Nhi Tran Thi, Jean-Claude Gerbedoen, et al.. Electronic and physico-chemical properties of nanometric boron delta-doped diamond structures. Journal of Applied Physics, 2014, 116, pp.083702. 10.1063/1.4893186 . hal-01058487

\section{HAL Id: hal-01058487 https://hal.science/hal-01058487}

Submitted on 3 May 2019

HAL is a multi-disciplinary open access archive for the deposit and dissemination of scientific research documents, whether they are published or not. The documents may come from teaching and research institutions in France or abroad, or from public or private research centers.
L'archive ouverte pluridisciplinaire $\mathbf{H A L}$, est destinée au dépôt et à la diffusion de documents scientifiques de niveau recherche, publiés ou non, émanant des établissements d'enseignement et de recherche français ou étrangers, des laboratoires publics ou privés. 


\section{Electronic and physico-chemical properties of nanometric boron delta-doped diamond}

structures.

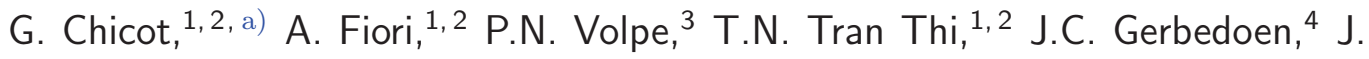
Bousquet,,${ }^{1,2}$ M.P. Alegre, ${ }^{5}$ J. C. Piñero, ${ }^{5}$ D. Araújo, ${ }^{5}$ F. Jomard, ${ }^{6}$ A. Soltani, ${ }^{4}$ J. C. De Jaeger, ${ }^{4}$ J. Morse,${ }^{7} \mathrm{~J}$. Härtwig, ${ }^{7}$ N. Tranchant, ${ }^{3}$ C. Mer-Calfati, ${ }^{3}$ J. C. Arnault, ${ }^{3} \mathrm{~J}$.

Delahaye, ${ }^{1,2}$ T. Grenet, ${ }^{1,2}$ D. Eon, ${ }^{1,2}$ F. Omnès, ${ }^{1,2}$ J. Pernot, ${ }^{1,2,8, b)}$ and E. Bustarret ${ }^{1,2}$

1) Univ. Grenoble Alpes, Inst. NEEL, F-38042 Grenoble France

${ }^{2)}$ CNRS, Inst. NEEL, F-38042 Grenoble France

${ }^{3)}$ CEA, LIST, Diamond Sensors Laboratory, F-91191 Gif-sur-Yvette,

France

4) IEMN, UMR-CNRS 8520, Avenue Poincaré, Université de Lille 1, 59652 Villeneuve d'Ascq, France

5) Dpto Ciencia de los Materiales, Facultad de Ciencias, Universidad de Cádiz, 11510 Puerto Real (Cádiz), Spain

${ }^{6)}$ Groupe d'Étude de la Matière Condensée (GEMaC), UMR 8635 du CNRS, UVSQ, 45 Avenue des États-Unis, 78035 Versailles Cedex,

France

7) European Synchrotron Radiation Facility, 38043 Grenoble,

France

8) Institut Universitaire de France, 103 boulevard Saint Michel, 75005 Paris, France 
Heavily boron doped diamond epilayers with thicknesses ranging from 40 to less than $2 \mathrm{~nm}$ and buried between nominally undoped thicker layers have been grown in two different reactors. Two types of [100]-oriented single crystal diamond substrates were used after being characterized by X-ray white beam topography. The chemical composition and thickness of these so-called delta-doped structures have been studied by secondary ion mass spectrometry, transmission electron microscopy and spectroscopic ellipsometry. Temperature-dependent Hall effect and four probe resistivity measurements have been performed on mesa-patterned Hall bars. The temperature dependence of the hole sheet carrier density and mobility has been investigated over a broad temperature range ( $6 \mathrm{~K}<T<450 \mathrm{~K}$ ). Depending on the sample, metallic or non-metallic behavior was observed. A hopping conduction mechanism with an anomalous hopping exponent was detected in the non-metallic samples. All metallic delta-doped layers exhibited the same mobility value, around $3.6 \pm 0.8 \mathrm{~cm}^{2} / \mathrm{Vs}$, independently of the layer thickness and the substrate type. Comparison with previously published data and theoretical calculations showed that scattering by ionized impurities explained only partially this low common value. None of the delta-layers showed any sign of confinement-induced mobility enhancement, even for thicknesses lower than $2 \mathrm{~nm}$.

a) Electronic mail: gauthier.chicot@neel.cnrs.fr

b) Electronic mail: julien.pernot@neel.cnrs.fr 


\section{INTRODUCTION}

The high breakdown electric field of diamond, its large carrier mobility and its exceptional thermal conductivity make it the ultimate semiconductor for high power and high frequency electronics. These features and the important progress that has been made recently in the fields of substrate fabrication, epilayer growth and doping control should in principle allow the development of new low loss electric switches ${ }^{1}$. However, the main problem for efficient power devices fabrication remains the large ionization energies of the doping impurities : 380 $\mathrm{meV}$ for the boron acceptor ${ }^{2,3}$ and $570 \mathrm{meV}$ for the phosphorus donor ${ }^{4}$. The high ionization threshold of the boron $p$-type ${ }^{2,3}$ results in a very low equilibrium carrier concentration at room temperature and thus in a very high material resistivity. Boron delta-doping ${ }^{5,6}$ has been proposed to overcome this problem, i.e. introducing a highly doped layer (metallic $\left.[B] \geq 5 \times 10^{20} \mathrm{~cm}^{-3}\right)$ stacked between two intrinsic layers, resulting in a conductive layer which combines a high mobility (due to a confinement-induced delocalisation of carriers away from the ionized impurities) with a large and almost $T$-independent carrier concentration (due to the metallic behavior). Calculations based only on the hole distribution in the Vshape potential suggested ${ }^{7}$ that partial delocalisation would occur for delta under $2 \mathrm{~nm}$. In the present work, boron top hat profiles with volume boron density of $[\mathrm{B}]=5 \times 10^{20} \mathrm{~cm}^{-3}$ and a width $\Delta z_{d}$ of $20 \mathrm{~nm}, 5 \mathrm{~nm}, 2 \mathrm{~nm}$ and $0.36 \mathrm{~nm}$ in a in a $1000 \mathrm{~nm}$ thick diamond (with $[\mathrm{B}]=10^{16} \mathrm{~cm}^{-3}$ ) are considered. The corresponding heavy holes valence band at 300 K plotted on Fig. 1 were calculated by solving Poisson equation and Schrödinger equation with Nextnano ${ }^{3}$ sofware developed by Walter Schottky Institute ${ }^{8,9}$. Hole effective masses given in Ref. 10 were used for calculations: heavy holes mass $m_{h h}^{*}=0.588 m_{0}$, light holes mass $m_{l h}^{*}=0.303 m_{0}$ and spin orbit split holes mass $m_{s o}^{*}=0.394 m_{0}$. In the case of the 5 $\mathrm{nm}$ and $20 \mathrm{~nm}$ thick delta-layers, 4 and 13 energy states corresponding to the heavy holes valence bands were respectively populated, far above the 3 first energy states plotted on Fig. 1. In addition, energy states corresponding to the two other valence sub-bands were also populated, but they are not plotted here, for the sake of clarity. To illustrate this, the densities of states (DOS) taking into account the three valences bands are plotted in figure 2. It can clearly be seen that for the thinnest layer, the DOS are step-like which is typical of $2 \mathrm{D}$ system, while for the widest layer $(20 \mathrm{~nm})$, as several levels are populated, the DOS

begins to be similar to the one of a 3D system (DOS $\propto \sqrt{E}$ as represented by the grey curve 

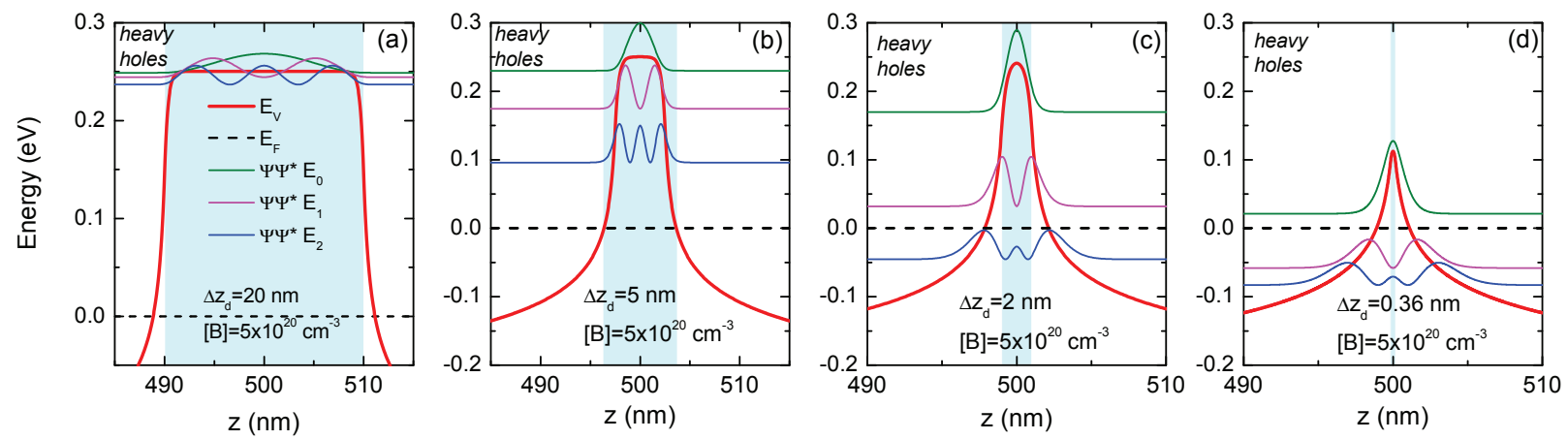

FIG. 1. Self-consistent calculated heavy holes valence band at $300 \mathrm{~K}$ corresponding to a boron top hat profile in a $1000 \mathrm{~nm}$ thick diamond (with $[\mathrm{B}]=10^{16} \mathrm{~cm}^{-3}$ ) with volume boron density of $[\mathrm{B}]=5 \times 10^{20} \mathrm{~cm}^{-3}$ and a width $\Delta z_{d}$ of (a) $20 \mathrm{~nm}$, (b) $5 \mathrm{~nm}$, (c) $2 \mathrm{~nm}$ and (d) $0.36 \mathrm{~nm}$. Each plot is focused on the carrier confined region and the self-consistent calculated free carrier distribution corresponding to the three first states $E_{0}, E_{1}$ and $E_{2}$ are plotted. Distribution densities $\left(\Psi(z) \Psi(z)^{*}\right)$ have been shifted according to their energy. The zero energy is the Fermi level (dashed line).

on Fig. 2).

Therefore, if holes are delocalized away from heavily doped regions, one can expect an enhancement of mobility which permits the use of delta-doped diamond for high frequency field effect transistors able to work at high temperature. Numerous works on diamond delta structures have been published recently by several groups, dealing both with fabrication and electrical characterization. Most of the electronic properties (sheet carrier density and mobility) of the delta structures have been determined by means of a field effect transistor $(\mathrm{FET})^{11-13}$. Temperature-dependent impedance spectroscopy measurements have also been performed to identify the different conduction paths in the stacked structures ${ }^{14,15}$. Hall effect combined to four probe resistivity measurements have also been used to evaluate the sheet density $\left(p_{S}\right)$ and the carrier mobility $\left(\mu_{H}\right)^{15-18}$. A low sheet carrier density $p_{S} \simeq 10^{13} \mathrm{~cm}^{-2}$ and a hole mobility $\mu_{H}=13 \mathrm{~cm}^{2} / \mathrm{V}$.s at room temperature in delta structures grown on [111]oriented diamond substrates were reported ${ }^{15}$, but unfortunately, no temperature dependence of $p_{S}$ and $\mu_{H}$ in delta structures were shown for the same samples. One of the recent works reported a very low mobility at low temperature $\left(\mu_{H} \sim 1 \mathrm{~cm}^{2} / \mathrm{V}\right.$.s at $\left.\mathrm{T}=100 \mathrm{~K}\right)$ and a high mobility at room temperature $\left(\mu_{H} \sim 900 \mathrm{~cm}^{2} / \mathrm{V} . \mathrm{s}\right)$. This was explained by a two carriertype $\operatorname{model}^{17}$, but the corresponding devices did not yield any improved performance.Scharpf 


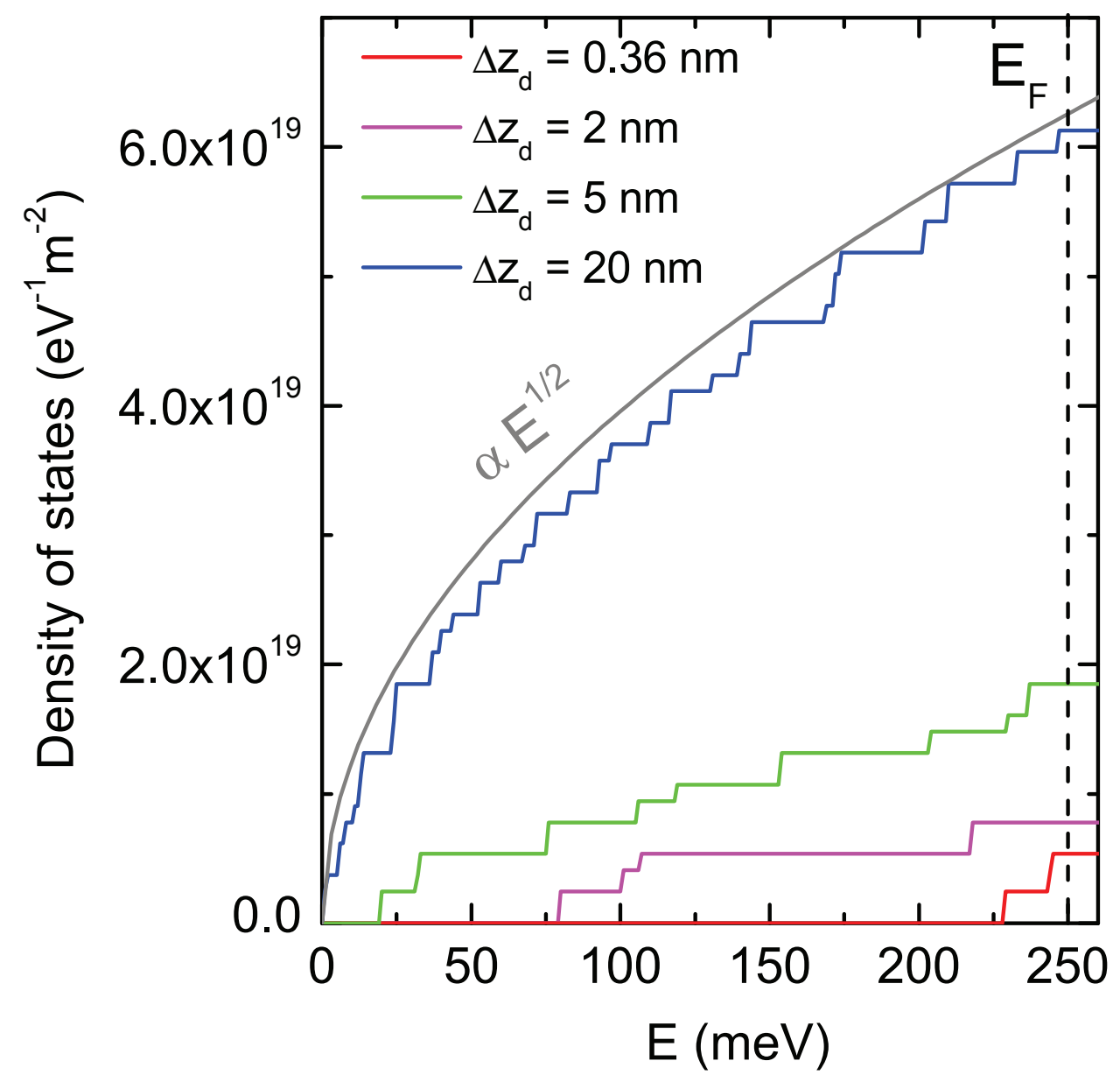

FIG. 2. Density of states (DOS) calculated for four delta layers $0.36 \mathrm{~nm}, 2 \mathrm{~nm}, 5 \mathrm{~nm}$ and 20 $\mathrm{nm}$ thick with $[B]=5 \times 10^{20} \mathrm{~cm}^{-3}$ corresponding to sheet carrier densities of $10^{15} \mathrm{~cm}^{-2}, 2.5 \times 10^{14}$ $\mathrm{cm}^{-2}, 10^{14} \mathrm{~cm}^{-2}$ and $1.8 \times 10^{13} \mathrm{~cm}^{-2}$ respectively. The DOS was calculated taking into account the three valence sub-bands.

et al $^{13}$ reported delta-layers with very low sheet carrier densities $\left(2\right.$ to $\left.4 \times 10^{13} \mathrm{~cm}^{-3}\right)$ also showing very low mobility ranging between $10^{-2} \mathrm{~cm}^{2} / \mathrm{V}$.s and $10^{-1} \mathrm{~cm}^{2} / \mathrm{V}$.s. A model based on hopping and tunneling processes was proposed to explain these mobility values. Some of us have shown in Ref. 18 that the analysis of the temperature dependence of $p_{S}(\mathrm{~T})$ and $\mu_{H}(\mathrm{~T})$ enable us to distinguish between each of the conduction paths (buffer/ high B-doped layer / cap-layer) which contribute to the measured (mixed) conductance. In the same work, it was demonstrated that even for delta layers only a couple of nanometers thick, the mobility enhancement expected if holes were quantum confined was not observed. 
The effective thickness of such thin delta-doped layers was deduced from their sheet carrier density, taking into account their apparent metallic behavior.

In this work, thin B-doped diamond layers embedded between non intentionally doped diamond layers were grown on two types of substrates using two different Microwave Plasmaenhanced Chemical Vapor Deposition (MPCVD) reactors and with different techniques. These layers were characterized by Secondary Ion Mass Spectroscopy (SIMS), Transmission Electron Microscopy (TEM) and ellipsometry. They were also assessed electrically by four probe and Hall effect measurements. The temperature dependence of the electrical transport properties are described and compared to the literature in order to evaluate in a reliable manner the carrier mobility and density in the hypothetical 2D hole gas. For metallic B-doped layers, the thickness deduced from the sheet carrier density is compared to the values deduced from SIMS and scanning TEM high angular annular dark-field (STEMHAADF) profiles. At low temperature, two types of conduction were detected in B-doped layers, metallic and non metallic, and a typical mobility value was measured for metallic samples. The measured mobilities are discussed in view of experimental values reported in the literature and theoretical calculated values. In section II, a description of the fabrication of samples and details about the experimental measurements will be given. Section III is dedicated to physico-chemical analysis of the delta layers by SIMS, TEM and ellipsometry. The temperature dependence of the hole sheet density and mobility are described and analyzed in section IV, separating the samples into two categories : metallic and non metallic. In section $(\mathrm{V})$ the experimental values of mobility are discussed further, before concluding remarks are given in section VI.

\section{EXPERIMENTAL DETAILS}

\section{A. SAMPLES DESCRIPTION AND GROWTH DETAILS}

Because of the nanometer-scale thickness of the delta-doped layers, particular attention was paid to the surface preparation of the commercial single crystal diamond substrates which had been grown at high pressure and high temperature (HPHT). Although quite successful in yielding subnanometer roughness ${ }^{19,20}$, the ion-implantation-related methods developed for that purpose did not deliver a surface upon which a suitably smooth epitaxial 
regrowth could be observed, probably because of the implantation-related residual defects. More traditional commercially available ultrapolishing methods ${ }^{21,22}$ giving 0.2 to $0.6 \mathrm{~nm}$ rms were employed. The process is known only in one case ${ }^{21}$ : first, a fine scaife polishing step, and then, an ion beam milling step, expected to remove the scaife-induced sub-surface damage. The miscut angle of the substrate surface relative to their nominal [100] orientation was measured to be $1.5 \pm 1^{\circ}$. The built quality of the substrates was further checked by white beam X-ray topography in the transmission performed at the BM05 beamline of the European Synchrotron Radiation Facility as detailed elsewhere ${ }^{23,24}$. As illustrated by fig. 3 in the case of the $\overline{2} 20$-reflection, the topography images revealed mostly dislocations, stacking faults along (111), and growth sectors of the HPHT stone from which the plate was cut. All layers were homo-epitaxially grown on Ib-type [100]-oriented $3 \times 3 \mathrm{~mm}^{2}$ diamond substrates (purchased from Sumitomo Electric), except for sample \#7, which was grown on the IIa-type [100]-oriented diamond substrate of higher structural quality (see Fig. 3).

\begin{tabular}{ccccccccc}
\hline \hline \multirow{2}{*}{ Sample } & \multirow{2}{*}{ Sub. } & Plasma & \multicolumn{2}{c}{$\mathrm{NiD}$ growth conditions } & \multicolumn{3}{c}{$p++$ growth conditions } \\
& & contact & $\mathrm{H}_{2}(\mathrm{sccm})$ & $\mathrm{CH}_{4} / \mathrm{H}_{2}$ & $\mathrm{O}_{2} / \mathrm{H}_{2}$ & $\mathrm{H}_{2}(\mathrm{sccm})$ & $\mathrm{CH}_{4} / \mathrm{H}_{2}$ & $\mathrm{~B} / \mathrm{C}(\mathrm{ppm})$ \\
\hline$\# 1$ & $\mathrm{Ib}(100)$ & point & 200 & $1 \%$ & $0.25 \%$ & 100 & $4 \%$ & 1500 \\
$\# 2$ & $\mathrm{Ib}(100)$ & point & 200 & $1 \%$ & $0.25 \%$ & 2000 & $0.5 \%$ & 6000 \\
$\# 3$ & $\mathrm{Ib}(100)$ & point & 200 & $1 \%$ & $0.25 \%$ & 2000 & $0.5 \%$ & 6000 \\
$\# 4$ & $\mathrm{Ib}(100)$ & point & 200 & $1 \%$ & $0.25 \%$ & 2000 & $0.5 \%$ & 6000 \\
$\# 5$ & $\mathrm{Ib}(100)$ & point & 200 & $1 \%$ & $0.25 \%$ & 2000 & $0.5 \%$ & 6000 \\
$\# 6$ & $\mathrm{Ib}(100)$ & point & 200 & $1 \%$ & $0.25 \%$ & 2000 & $0.5 \%$ & 6000 \\
$\# 7$ & $\mathrm{IIa}(100)$ & surface & 200 & $0.75 \%$ & $0.32 \%$ & 2000 & $0.5 \%$ & 6000 \\
$\# 8$ & $\mathrm{Ib}(100)$ & surface & 200 & $0.75 \%$ & $0.32 \%$ & 2000 & $0.5 \%$ & 6000 \\
$\# 9$ & $\mathrm{Ib}(100)$ & point & 200 & $1 \%$ & $0.25 \%$ & 2000 & $0.5 \%$ & 6000 \\
$\# 10$ & $\mathrm{Ib}(100)$ & - & 400 & $1 \%$ & $0.25 \%$ & 400 & $0.6 \%$ & 21400 \\
\hline \# $\mathrm{TdL1}$ & $\mathrm{Ib}(100)$ & surface & 200 & $0.75 \%$ & $0.32 \%$ & 2000 & $0.5 \%$ & 6000 \\
$\# \mathrm{TdL2}$ & $\mathrm{Ib}(100)$ & surface & 200 & $0.75 \%$ & $0.32 \%$ & 2000 & $0.5 \%$ & 6000 \\
\hline \hline
\end{tabular}

TABLE I. (100) substrate-types, and growth conditions for samples used in this work.

Twelve boron doped samples were grown by MPCVD. Samples \#1 to \#9 were grown on a modified vertical silica tube (so-called "NIRIM-type") reactor, where the volume was 
reduced and an ultra-fast gas switching system implemented. A well-controlled in situ plasma etch process was also developed ${ }^{26}$. Sample \#10 was grown in an all-metal reactor where a silica gas injector had been introduced in the vicinity of the sample surface to add suddenly Trimethylboron molecules in the gas mixture ${ }^{27,28}$. An injection time of 90 seconds was used to grow a thin $p^{++}$layer. The reproducibility of this process was previously reported in ref. ${ }^{29}$ as well as the stability of the plasma ball. The injector was sufficiently far from the plasma ball not to be etched but close enough to allow a quick change of the growth parameters $^{29}$. Except for two double delta-layer structures (\#TdL1 \#TdL2), all samples consisted of a highly $p$-doped $\left([B] \geq 5 \times 10^{20} \mathrm{~cm}^{-3}\right.$, labelled $p^{++}$in the following) layer of thickness $<35 \mathrm{~nm}$ grown on a thick non intentionally doped (NiD) buffer layer suited for high mobility transport ${ }^{3,28}$ and capped by another thin NiD cap layer (of thickness 30 to 65 nmin) with similar nominal properties. Additional multilayers containing several delta layers were also grown to investigate growth and etch rates, these were characterized by ellipsometry $^{32}$ and SIMS.
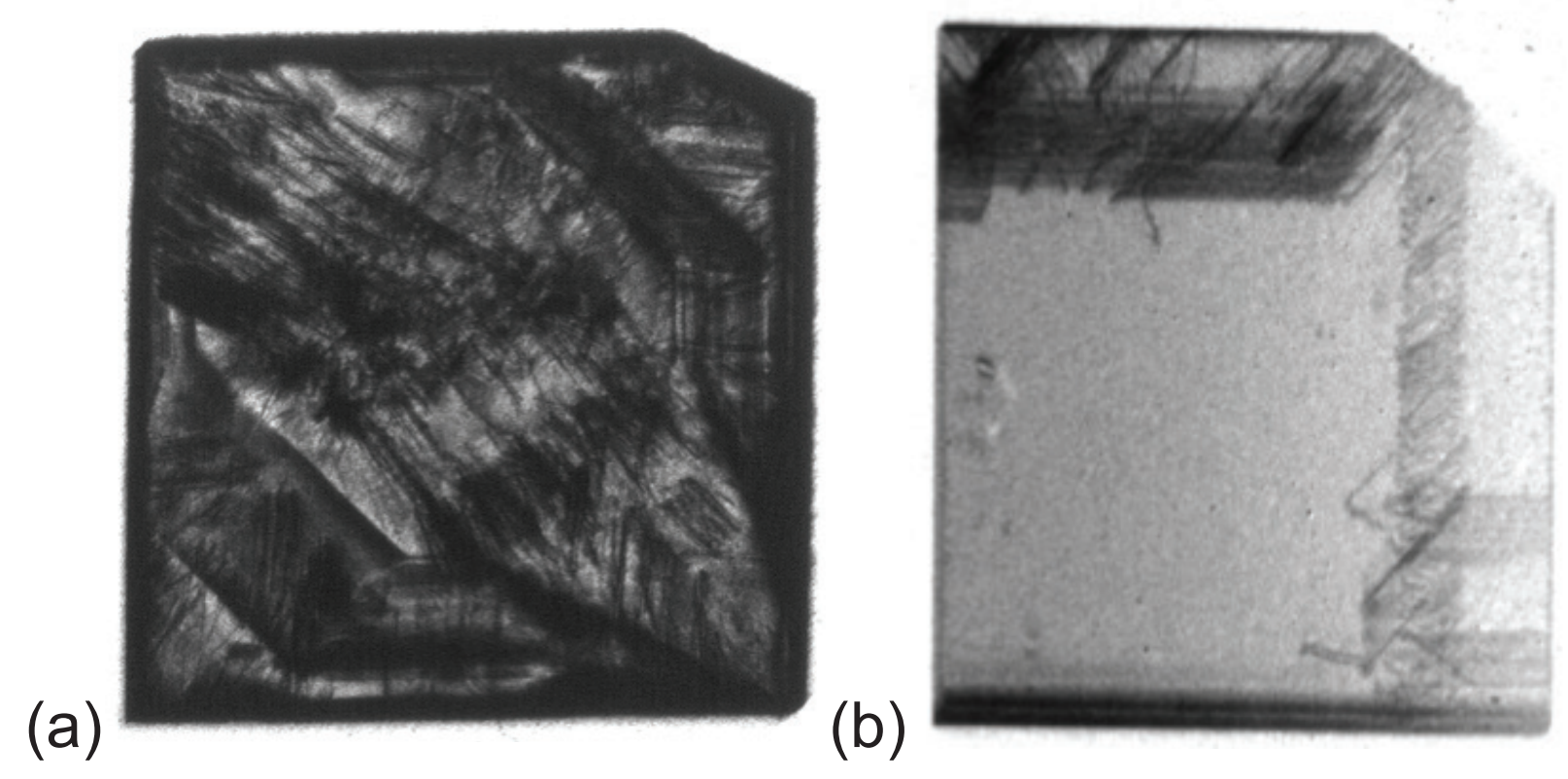

FIG. 3. X ray Topography (white beam) pictures for the 220 -reflection of (a) Ib and (b) IIa diamond substrate. Both dislocations and stacking faults are readily observable.

The NIRIM type reactor allowed accurate positioning of the sample with respect to the plasma glow discharge ball. In this work, two different vertical positions were used for each 
sample as described in table I: i) "surface contact", where the top surface of the sample was immersed within the plasma ball and ii) "point contact", where the center of the top of the sample was lowered to the edge of the plasma ball. Two sets of NiD mixtures were used to grow nominally undoped regions (buffer and cap layers) of the delta-structures. NiD conditions for each sample grown in the NIRIM-type reactor are given in table I. The first $\mathrm{NiD}$ mixture composed of $\mathrm{CH}_{4} / \mathrm{O}_{2} / \mathrm{H}_{2}(1 \%, 0.25 \%, 0.9875$ molar) has been shown to be suited for high mobility transport ${ }^{3}$. This recipe was next improved by modifying the gas ratio such as $\mathrm{CH}_{4} / \mathrm{O}_{2} / \mathrm{H}_{2}(0.75 \%, 0.32 \%, 0.9893$ molar) to favour lateral growth (i.e. vertical growth rate lower than lateral growth rate) in order to reduce the surface roughness, particularly before growing the delta-layer. The heavily doped $\left(p^{++}\right)$delta layers of samples \#2 to \#9 and \#TdL1 and \#TdL2 were grown in a 50 Torr $\mathrm{CH}_{4} / \mathrm{B}_{2} \mathrm{H}_{6} / \mathrm{H}_{2}$ mixture (with $\mathrm{CH}_{4} / \mathrm{H}_{2}=0.5 \%$ and $\mathrm{B} / \mathrm{C}=6000 \mathrm{ppm}$ ) flowing at $2000 \mathrm{sccm}$ before being etched in situ at the same total pressure in an $\mathrm{O}_{2} / \mathrm{H}_{2}$ mixture $(0.25 \%, 0.9975$ molar) flowing at $200 \mathrm{sccm}$. They were then covered by a thin cap layer grown in the same gas mixture and under the same conditions as the initial buffer layer. Note that the plasma was kept on throughout the whole process, as described elsewhere ${ }^{26}$. The role of the etching step was to reduce the highly $p$-doped layer thickness and to improve the sharpness of the B-doping profile at the top interface of the highly doped layer. The in situ etching also reduced the residual boron level in the NiD layers due to memory effects in the reactor. Between each step, a 3 min $2000 \mathrm{sccm} \mathrm{H}_{2}$ rinsing step was introduced in order to wash the reaction chamber and to start the next step with minimal residual species. Consequently, a typical sequence for growing a delta-structure was $\mathrm{NiD}(200 \mathrm{sccm}) / \mathrm{H}_{2}(2000 \mathrm{sccm}) / p^{++}(2000 \mathrm{sccm}) / \mathrm{H}_{2}(2000 \mathrm{sccm}) /$ etching $(200 \mathrm{sccm}) / \mathrm{H}_{2}$ (2000 sccm) / NiD (200 sccm). Sample \#10 was grown using an injection tube in a metallic reactor. Gas flow was kept constant at $400 \mathrm{sccm}$ for the growth of this delta structure. Growth in this metallic reactor with a gas injector was optimized for an injection time of 90 sec. for the $p^{++}$layer. No etching was needed to obtain sharp interfaces between the $p^{++}$and the NiD layers ${ }^{29}$.

Two samples \#TdL1 and \#TdL2, composed of two delta-layers each, were also grown in the same reactor as sample \#2 to \#9. One (\#TdL1) was dedicated to TEM measurements while the other was intended for SIMS analysis. These two-delta-layers structures were identical, except for the thickness of the NiD spacers. A thicker cap layer was grown for the sample intended for TEM analysis. The two delta-layers of each TdL, were grown using 
the same recipe as samples \#2 to \#9 before being etched in situ, under the NiD growth conditions given in table I. The same $p^{++}$growth duration was used for both samples, but the etching times differed in order to obtain two different $p^{++}$thicknesses.

\section{B. PHYSICO-CHEMICAL CHARACTERIZATIONS}

SIMS measurements were performed using $\mathrm{O}_{2}^{+}$as primary ions at $1 \mathrm{keV}$ and $\mathrm{Cs}^{+}$at 15 $\mathrm{keV}$ in a Cameca ims $7 \mathrm{f}$ system, with collection of the negative secondary ${ }^{11} \mathrm{~B}$ and ${ }^{12} \mathrm{C}$ ions or their compounds. STEM-HAADF micrographs were taken in the scanning mode using a Jeol 2010 TEM with a $200 \mathrm{kV}$ beam, $0.7 \mathrm{~nm}$ probe size and $8 \mathrm{~cm}$ camera length. The sample was prepared with a Quanta 200 3D focused ion beam, with a final thickness of approximately $70 \mathrm{~nm}$ as detailed elsewhere ${ }^{30}$. Spectroscopic ellipsometry measurements were made taking advantage of the difference in refractive indices of $\mathrm{NiD}$ and $\mathrm{p}^{++}$diamond: these were performed from 250 to $1000 \mathrm{~nm}$ in air using a Woollam M-2000 ellipsometer. The experimental spectra were fitted to numerical simulations as described elsewhere ${ }^{32}$ in order to determine the respective thicknesses of the $\mathrm{NiD}$ and $p^{++}$layers present in the alternating $\mathrm{NiD} / p^{++} / \mathrm{NiD}$ multilayers.

\section{ELECTRICAL CHARACTERIZATION METHODS}

Hall bars were fabricated on samples \#2 to \#10 to perform Hall effect and four probe resistivity measurements following the chronology schematized on figure 4. For samples $\# 2$ to \#6 and \#9, the bars were delineated by $\mathrm{O}_{2}$ plasma etching of the diamond around a mesa. Ti/Pt/Au pads were deposited by evaporation and annealed at $1025 \mathrm{~K}$ (not any boron diffusion is expected at such temperature ${ }^{31}$ ) under vacuum $<10^{-8}$ mbar during 30 min in order to obtain low resistance ohmic contacts. For sample \#7, \#8 and \#10, the samples were chemically cleaned and oxidized by a $\left(\mathrm{H}_{2} \mathrm{SO}_{4}, \mathrm{HNO}_{3}\right)$ mixture at $450 \mathrm{~K}$ for 3 hours. All patterns were made by electron beam lithography. Marks and mesa were defined and a $150 \mathrm{~nm}$ thick Ni mask was deposited by evaporation. An ICP plasma etching with $\mathrm{Cl}_{2} / \mathrm{Ar}$ $(40 / 25 \mathrm{sccm})$ mixture was then used to define the mesa with $200 \mathrm{~nm}$ depth. A Ti/Pt/Au $(30 / 50 / 140 \mathrm{~nm})$ metal-layer stack was deposited by evaporation to form the ohmic contact followed by annealing at $1025 \mathrm{~K}$ for 2 hours under a vacuum of $3 \times 10^{-7}$ mbar. Finally, 


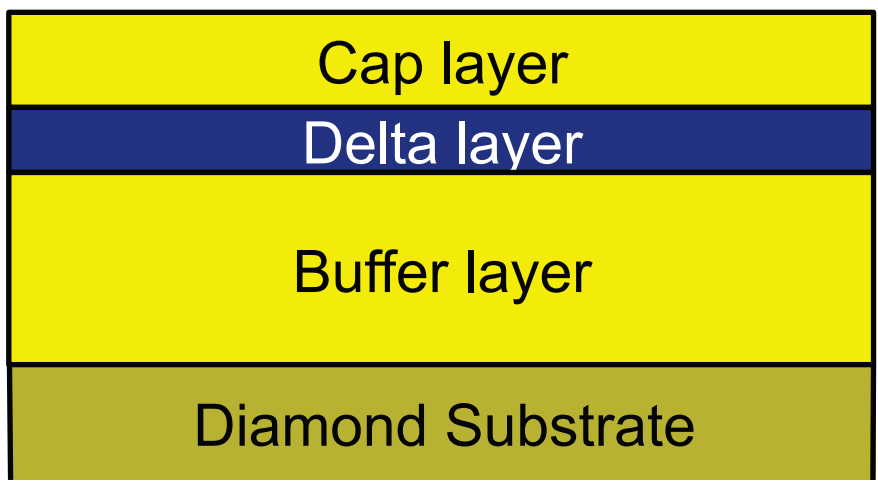

(a) Delta structure on diamond substrate

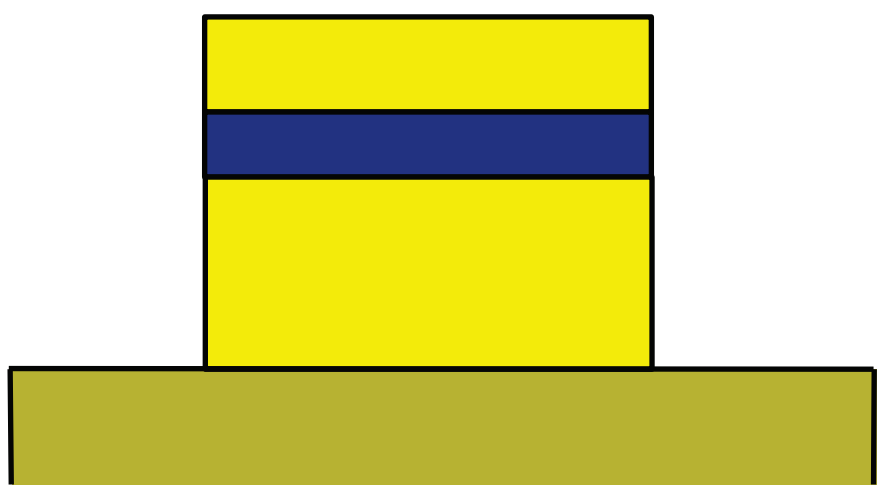

(b) Mesa structure delimited by an etching step

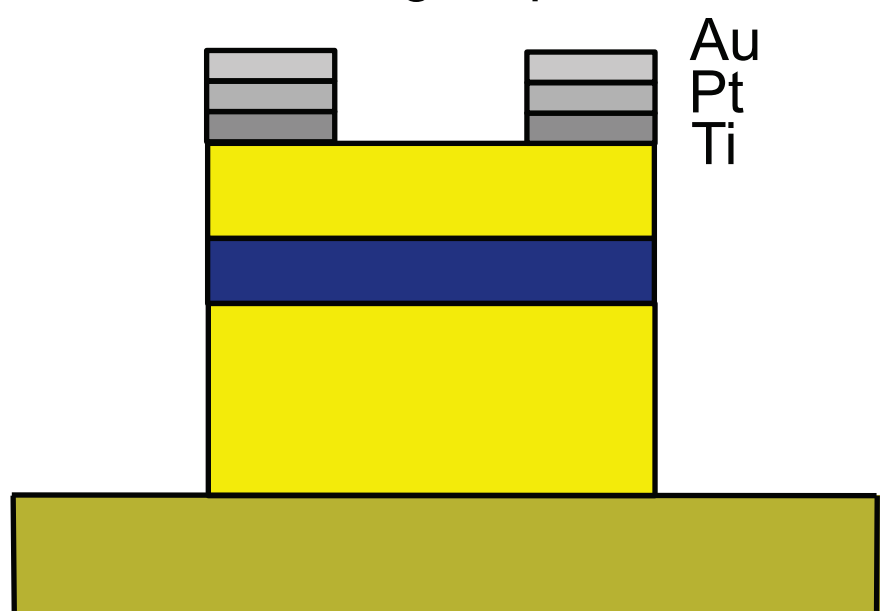

(c) Deposition and annealing of ohmic contacts

FIG. 4. Schematics of Hall bars fabrication steps from side view of sample. 
thick Ti/Au (100/400 nm) pads were deposited by evaporation to enable characterization of the sample.

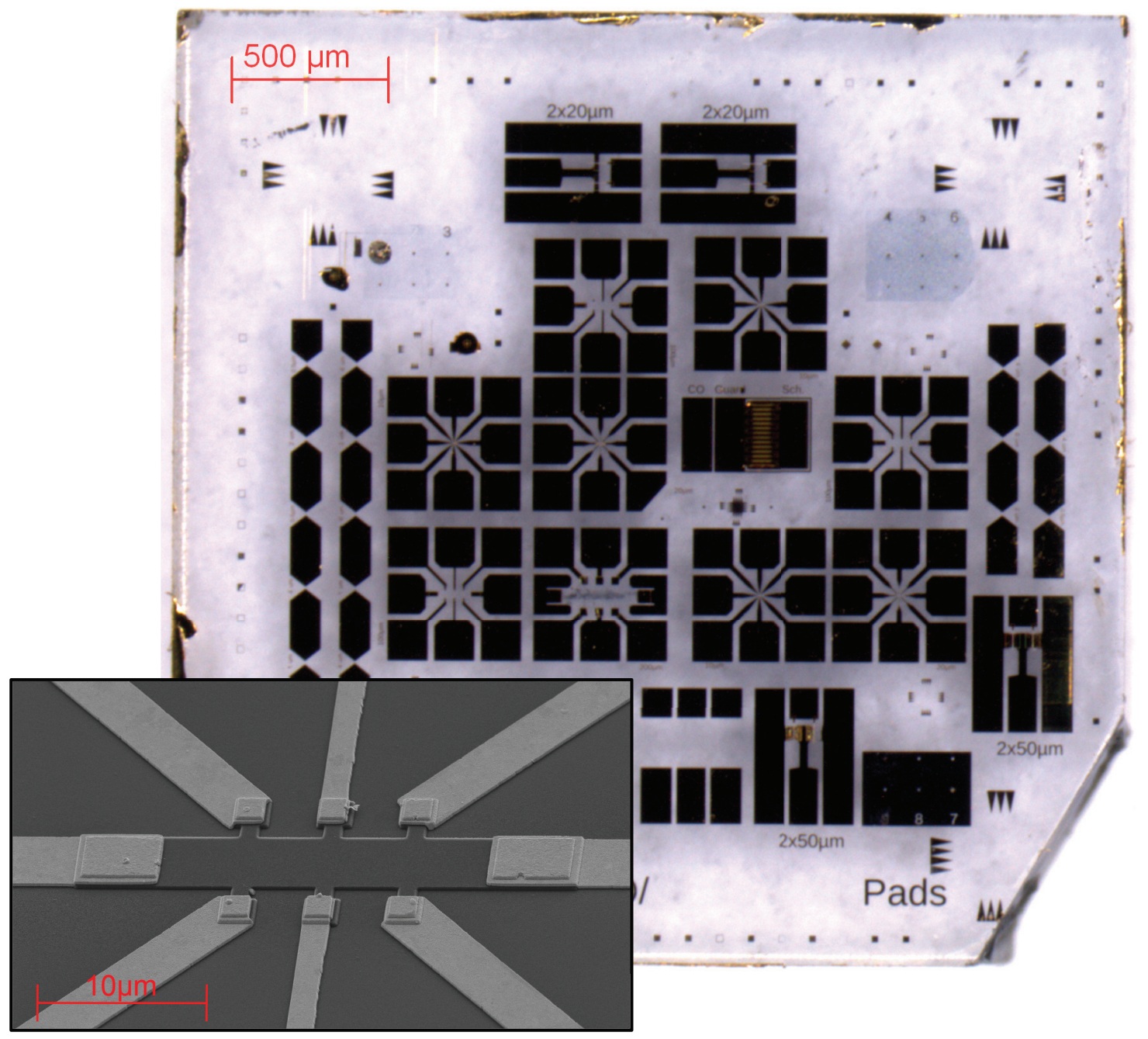

FIG. 5. Whole surface picture of sample \#3 where Hall bars of different sizes (from $10 \mu \mathrm{m}$ to 200 $\mu \mathrm{m})$ can be seen. The inset is a Scanning Electron Microscopy image of a $20 \mu \mathrm{m}$ long Hall bar.

Hall effect measurements were performed under well controlled conditions (vacuum $<10^{-4}$ mbar) between $6 \mathrm{~K}$ and $450 \mathrm{~K}$. Ohmicity of contacts was checked by current-voltage $I(V)$ measurements over the whole range of temperature. Hall effect measurements were carried out with a $d c$ magnetic field $\mathbf{B}$ of $0.8 \mathrm{~T}$ in the standard configuration (i.e. $\mathbf{B}$ parallel and $j$, the current density, perpendicular to the growth axis [100]). On samples \#2 to \#6 and \#9, Hall bars of $500 \mu \mathrm{m}$ length were fabricated, while on sample \#7 to \#8 and \#10, Hall bar lengths varied from $10 \mu \mathrm{m}$ to $200 \mu \mathrm{m}$ as shown in Fig. 5. Hall measurements shown 


\begin{tabular}{ccccccc}
\hline \hline Recipe & $\begin{array}{c}\mathrm{H}_{2} \\
(\mathrm{sccm})\end{array}$ & $\begin{array}{c}\mathrm{CH}_{4} \\
(\%)\end{array}$ & $\begin{array}{c}\mathrm{O}_{2} \\
(\%)\end{array}$ & $\begin{array}{c}\mathrm{B} / \mathrm{C} \\
(\mathrm{ppm})\end{array}$ & $\begin{array}{c}\text { Growth rate } \\
\left(\mathrm{nm} . \mathrm{min}^{-1}\right)\end{array}$ & $\begin{array}{c}\text { Etch. rate } \\
\left(\mathrm{nm} . \mathrm{min}^{-1}\right)\end{array}$ \\
\hline $\mathrm{NiD} \mathrm{1}$ & 200 & 1 & 0.25 & & $8^{\mathrm{a}}$ & \\
$\mathrm{NiD} 2$ & 200 & 0.75 & 0.32 & & 6.6 & \\
$p^{++}$ & 2000 & 0.5 & & 6000 & 6.7 & 0.45 \\
etching & 200 & & 0.25 & & & 0.3 \\
$\mathrm{H}_{2}$ & 2000 & & & & & \\
\hline \hline
\end{tabular}

TABLE II. Etching and growth rate for different gas mixtures used in the NIRIM type reactor for growing (and etching) delta-structures in the surface contact mode. To one exception, all rate values were measured by ellipsometry.

${ }^{a}$ Measured by SIMS

in this paper were performed on one Hall bar per sample (except on sample \#5 where two Hall bars were investigated in order to check the homogeneity of the sheet carrier density). Sheet resistance (four terminated probe measurements) was investigated for different Hall bar sizes on two samples (sample \#7 and \#8) grown on different types of substrates.

\section{GROWTH RATE AND THICKNESS EVALUATION}

In order to fabricate very thin delta-layers, the growth and etch rates must both be known. For the different plasma mixtures used in this work, these rates were evaluated using ellipsometry, and in some cases, SIMS as reported in table II. As suggested in a previous work $^{26}$, it has been found that the $2000 \mathrm{sccm} \mathrm{H}_{2}$ rinsing step was also an etching step with a non negligible etching rate. Typical nanometric scale delta layers were introduced in sample \#TdL1 and \#TdL2. The thinner delta layer labeled $\alpha$ was etched for a longer time than the layer labeled $\beta$.

Figure 6 displays STEM-HAADF images of \#TdL1 where the two delta layers $\alpha$ and $\beta$ can be distinguished by a darker contrast than the NiD region. The grey scale "noise" in this STEMHAADF image is attributed to a residual amorphous overlayer and to local lamella thickness variations $^{30}$. As can be seen in Fig. 6 (b) and (c), the nanometric scale was reached. The normalized STEM-HAADF intensity integrated over the width of the dash lined rectangle 
in Fig. 6 (a) was plotted in Fig. 7 (a). The thickness of each delta layer was estimated at the Full Width at Half Maximum (FWHM) of the STEM-HAADF signal and was found to be $2.2 \mathrm{~nm}$ for delta $\alpha$ and $3.2 \mathrm{~nm}$ for delta $\beta$. As the STEM beam spot size was around 0.7 $\mathrm{nm}$, the doping transition sharpness of the profile is shown here to be in the same size range. Moreover, the ground of the profile was not reached. This means that the real thickness is probably even lower than that deduced above. Note that here a quantitative boron doping profile deduced from such profiles, as recently published using a STEM-HAADF based method ${ }^{30}$, was not derived as this ground value of the profile was not reached so that the inferred doping maximum value would be erroneous. An alternative would be to use atomic resolution STEM-HAADF with a much lower beam spot size, but the difficulties in preparing sufficiently thin FIB-mchined lamella without any amorphization prevented us from obtaining the required atomic contrasts. Indeed, as carbon is a relatively light material, the ion beam spreading inside the material during the high energy bombardment of the sample preparation induces a relatively thick superficial damage layer, even when using last cleaning steps at $1 \mathrm{keV}$. Similar preparations on Si samples or on metal contact on diamond resulted in clearer high resolution TEM (HREM) observations that showed a transition from atomic related contrasts in the metallic contact to amorphous-like in the diamond.

A SIMS profile of sample \#TdL2 where the two delta layers were grown under the same conditions as \#TdL1 is shown in Fig. 7 (b). In the case of nanometric thick and heavily boron doped layers $\left([\mathrm{B}]>10^{20} \mathrm{~cm}^{-3}\right)$, it has been shown that ion mixing and low spatial resolution can lead to erroneous values of the thickness and doping levels on the raw SIMS profile $^{29}$, unless the Depth Resolution Function (DRF) is known and convolved with the SIMS data to obtain a corrected profile ${ }^{33}$. A boron depth profile $\left(15 \mathrm{keV}, \mathrm{Cs}^{+}\right.$as primary ions at an incidence angle of $27^{\circ}$ ) was fitted using the DRF determined from experiment with isotopic carbon substitution in another delta layer as described in Refs. 33 and 34. The results confirmed the expected values for the thickness $(\sim 1 \mathrm{~nm}$ for $\alpha$ and $\sim 2 \mathrm{~nm}$ for $\beta)$ and the atomic concentration for both boron-delta-layers $\left([\mathrm{B}]=1.2 \times 10^{21} \mathrm{~cm}^{-3}\right)$. As the depth sampling of SIMS analysis was on the same order as the delta layers thickness, it was not possible to evaluate the interface thickness. This point may explain the difference between thickness values determined by STEM-HAADF at FWHM and by SIMS analysis. To be more accurate, SIMS analysis should be performed with lower energy primary ions 
such as those shown in Fig. 7 (b). Unfortunately, the DRF was not determined for such conditions.
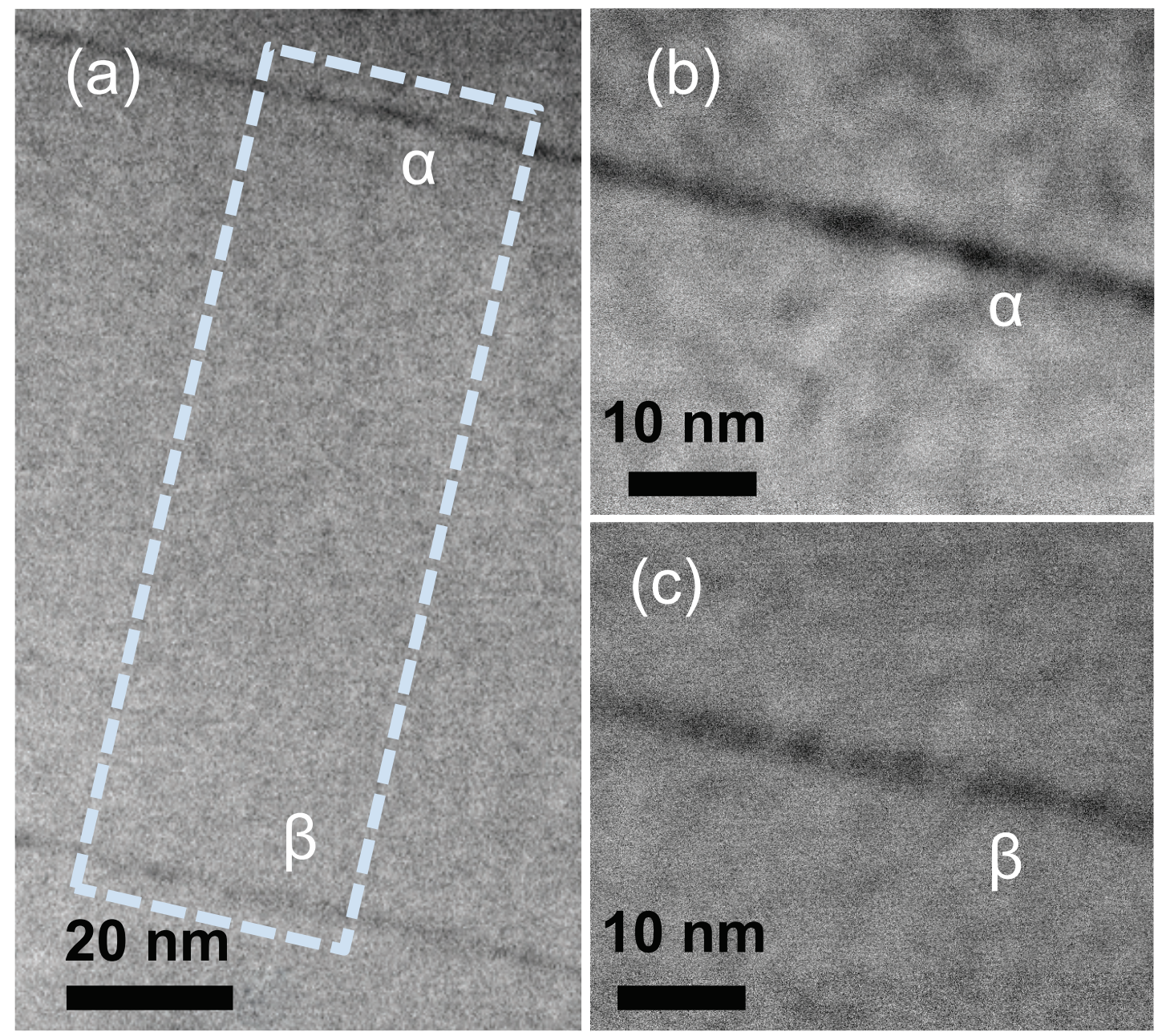

FIG. 6. STEM-HAADF delta-layers in [100] zone axis (a) and STEM-HAADF of both top (b) and bottom (c) layers.

\section{HALL EFFECT AND SHEET RESISTANCE RESULTS}

Hall effect and four probe sheet resistance measurement are two complementary tools to determine electronic transport properties of delta layer structures. Assuming that the conduction occurs only in the rectangular profile delta layer of thickness $d$, four probe sheet resistance and Hall effect measurements allow us to determine respectively $i$ ) the sheet resistance $R_{s}=\rho / d$ where $\rho$ is the resistivity and $i i$ ) the sheet carrier density $p_{S}=p . d$ where 

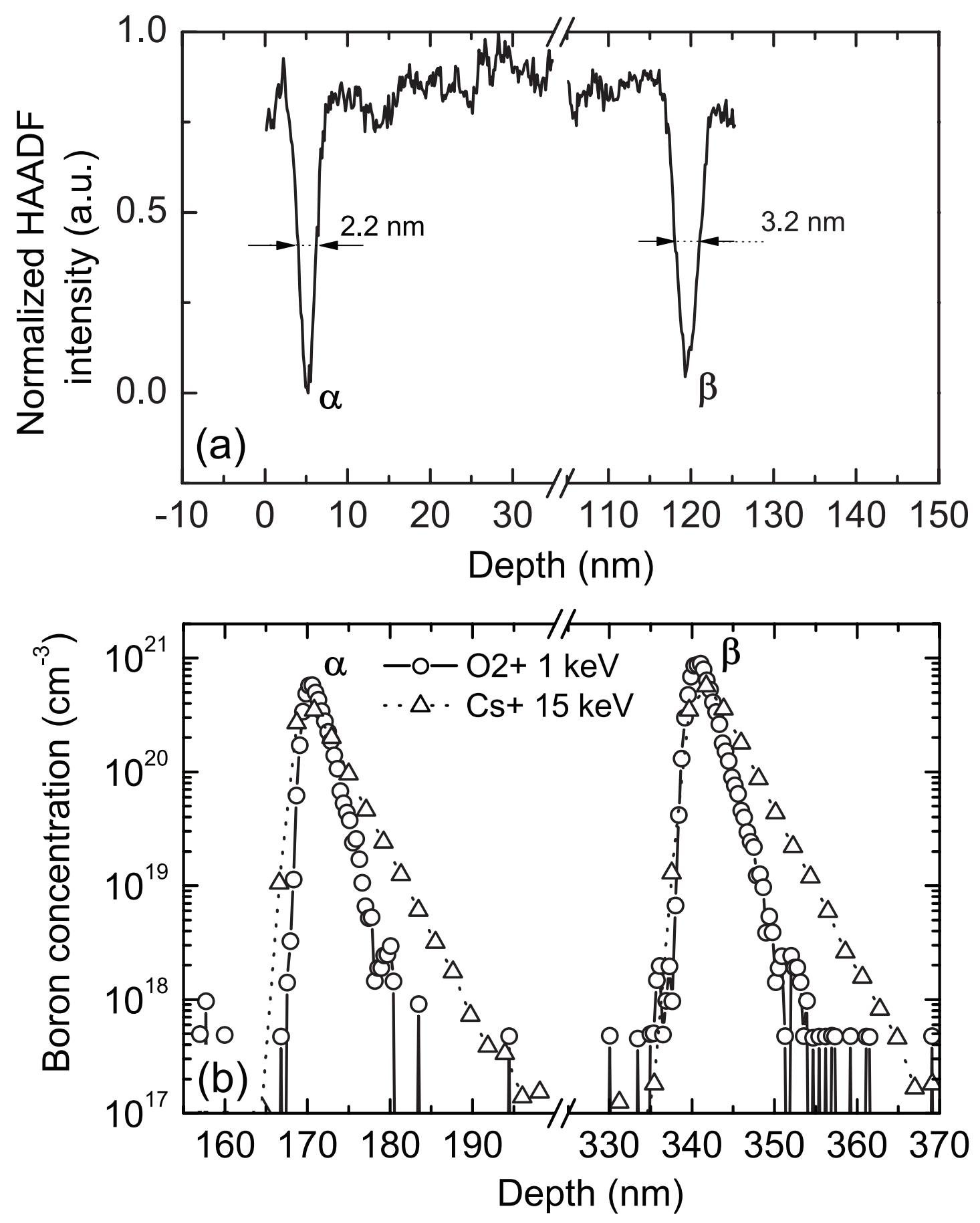

FIG. 7. (a) Normalized STEM-HAADF intensity versus depth of 2 delta-layers (\#TdL2) where the top layer $\alpha$ and bottom layer $\beta$ show respectively a $2.2 \mathrm{~nm}$ and $3.2 \mathrm{~nm}$ thickness in FWHM, and (b) boron profile obtained by SIMS analysis of 2 delta-layers grown under the same conditions (\#TdL1). 


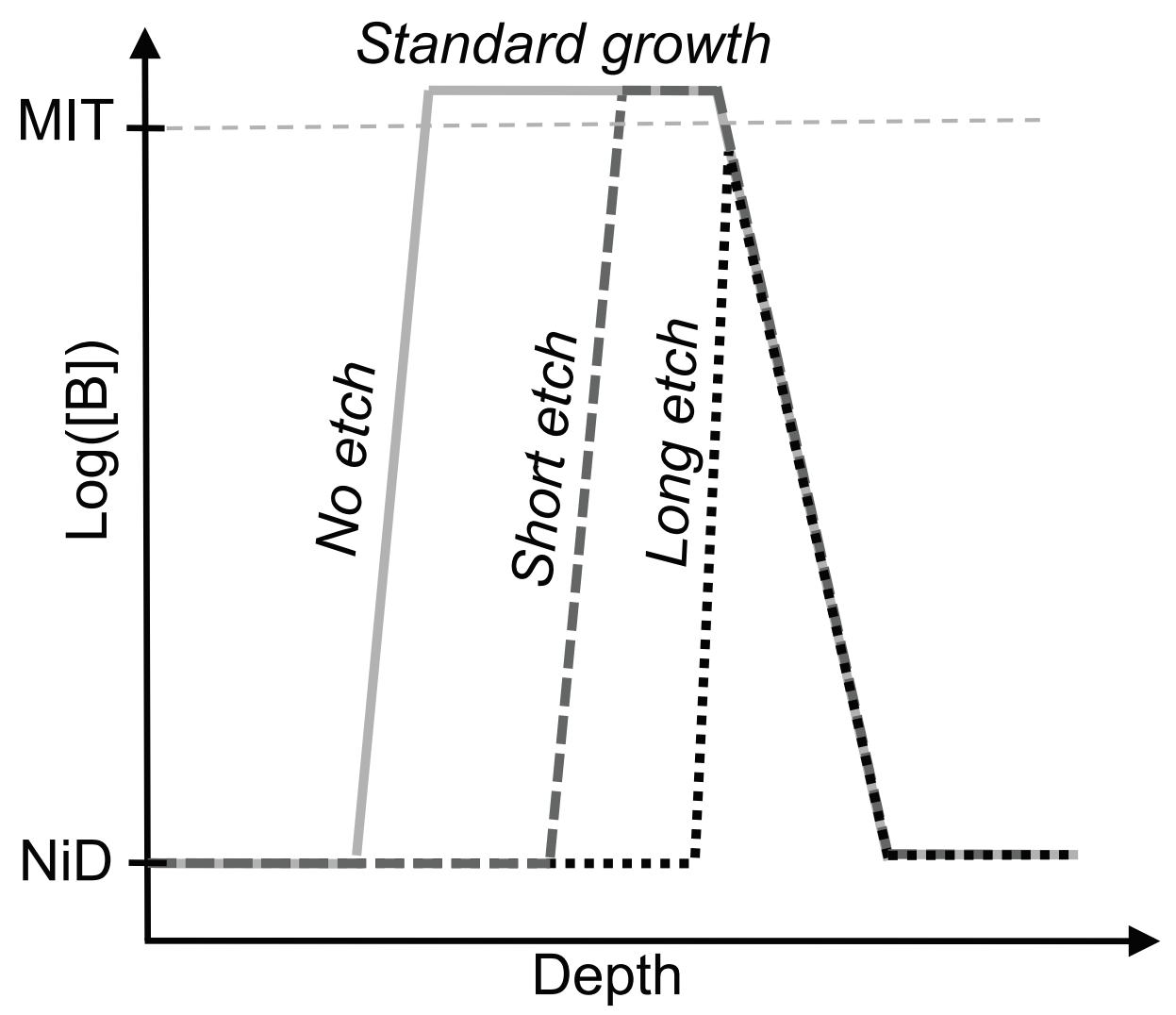

FIG. 8. Different schematical boron concentration profiles showing how the etching step duration affects the thickness and the peak boron concentration.

$p$ is the carrier density (in $\mathrm{cm}^{-3}$ ). From these two physical values we can determine the mobility independently of the delta layer thickness $d$ as $\mu=1 /\left(q \cdot p_{S} \cdot R_{S}\right)$.

The same doping level was aimed for during the $p^{++}$growth step as all samples (except \#1 and $\# 10)$ were grown with the same recipe. This $p^{++}$layer was etched to reduce the thickness of the final layer (thickness values are discussed in section IV B 3). Moreover, the final boron concentration peak of a delta layer may also be affected by the etch step duration ${ }^{26}$ as illustrated in figure 8. Therefore, some of the delta layers may had a boron concentration under the critical value for the metal to insulator transition ${ }^{35}$ (MIT) and be non metallic. From the measurements of the electrical properties as a function of temperature, the samples analyzed in this work could be classified into two categories depending on their sheet resistance behaviour: $A$ ) non metallic conduction behaviour samples with zero conductance at low temperature and which exhibit a sheet resistance, sheet carrier density and mobility 
varying with the temperature and $B$ ) metallic conduction behavior samples with a finite conductance at low temperature, and which have a quasi constant sheet resistance, sheet carrier density and mobility over the whole temperature range.

Indeed, one of the interesting properties of boron doped diamond is the evolution from a semiconductor to a metal when increasing the boron level above the critical concentration $N_{\text {crit }}=5 \times 10^{20} \mathrm{~cm}^{-3}$ which was determined for thick epilayers ${ }^{35}$. In the following, it will be assumed that the boron ionization energy $E_{a}$ decreased following the same trend as in thick epilayers $^{36}$ (i.e. $\left.E_{a}=E_{a 0}\left(1-\left([B] / N_{\text {crit }}\right)^{1 / 3}\right)\right)$.

\section{A. NON METALLIC DELTA LAYERS}

As shown in Fig. 9, the sheet resistance of samples \#6 and \#10 was larger than $10^{8} \Omega$ for $T<30 \mathrm{~K}$ and increased with decreasing temperature. The delta layer of sample \#6 is expected to be very thin because of a longer in-situ etch step after $p^{++}$layer growth as described in Ref. 26. Thus, for a fixed B/C ratio in the gas phase (here 6000 ppm), two parameters can influence the thickness of the resulting layer: the $p^{++}$growth step time and the etching step duration. As illustrated by figure 8 , if the $p^{++}$step is too short and/or

the etching step too long, the maximum doping level of the resulting $p^{++}$layer may be reduced. In this case, the boron concentration may become lower than $N_{\text {crit }}$ for the metallic to insulator transition and the activation energy $E_{a} \neq 0$. The doping level $[\mathrm{B}]$ of these samples may then be just below the critical concentration $\left([B]<N_{\text {crit }}\right)$, inducing a MIT. This is a doping range where the conductivity is known to be dominated by hopping mechanisms ${ }^{37}$ between localized states instead of conventional valence band (delocalized states) conduction limited by scattering mechanisms. Indeed, the sheet resistance of these two samples can be fitted by (see full lines in figure 10):

$$
R_{S}(T)=R_{0} \cdot \exp \left(\frac{T_{0}}{T}\right)^{x}
$$

where $x$ is the hopping exponent. The good agreement between the experimental data and this model confirms the hopping conduction and the zero conductance at low temperature $\left(G_{S} \rightarrow 0 \mathrm{~S}\right.$ for $\left.T \rightarrow 0 \mathrm{~K}\right)$. These observations corroborate the non metallic conduction (with $\left.[\mathrm{B}]<N_{\text {crit }}\right)$ suggested above. As the Hall voltage was below the detection limit value for $T<$ 200K, sheet carrier densities and mobilities are shown only for $T>200 \mathrm{~K}$ on figure 9 (b) 

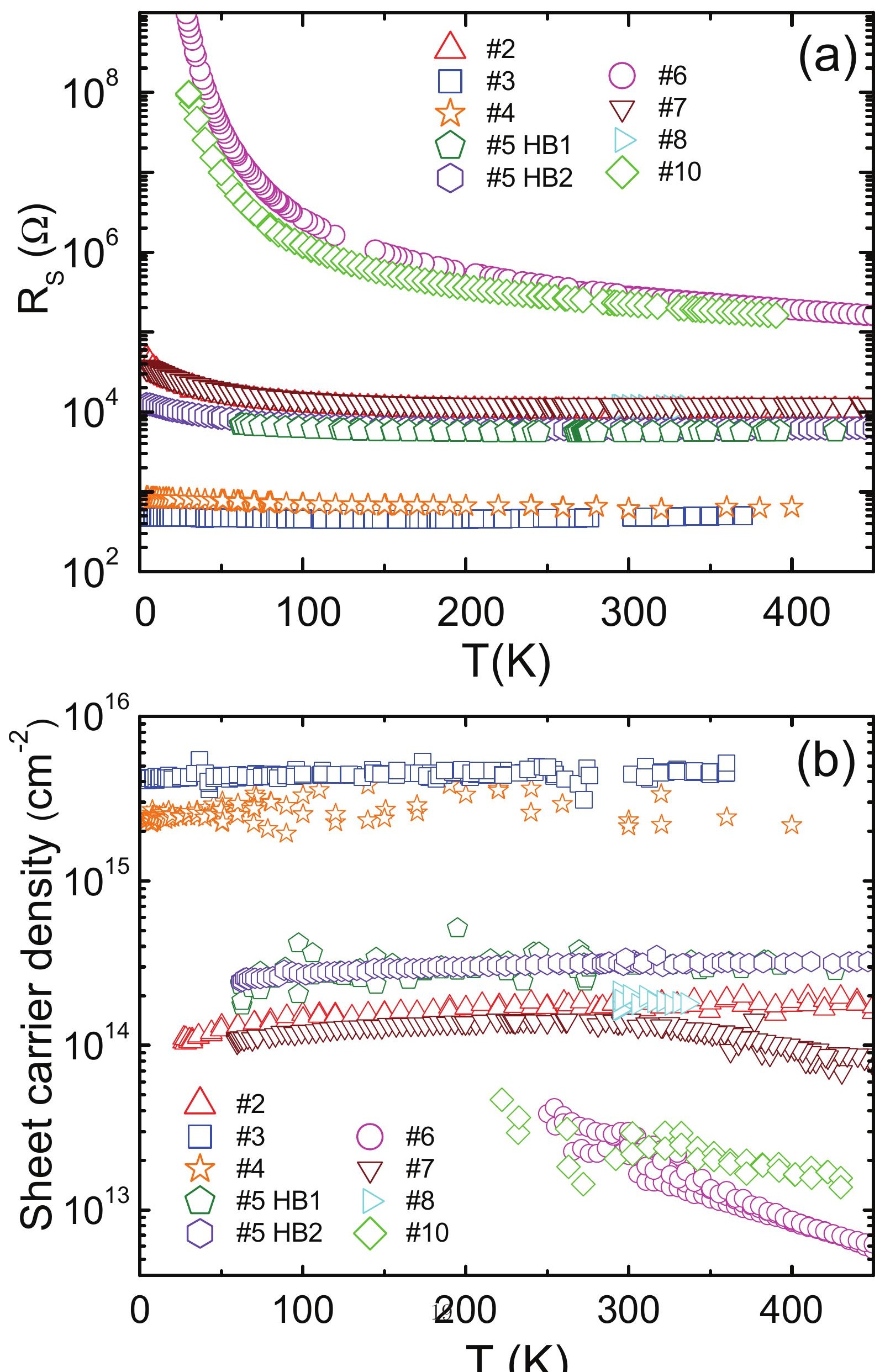


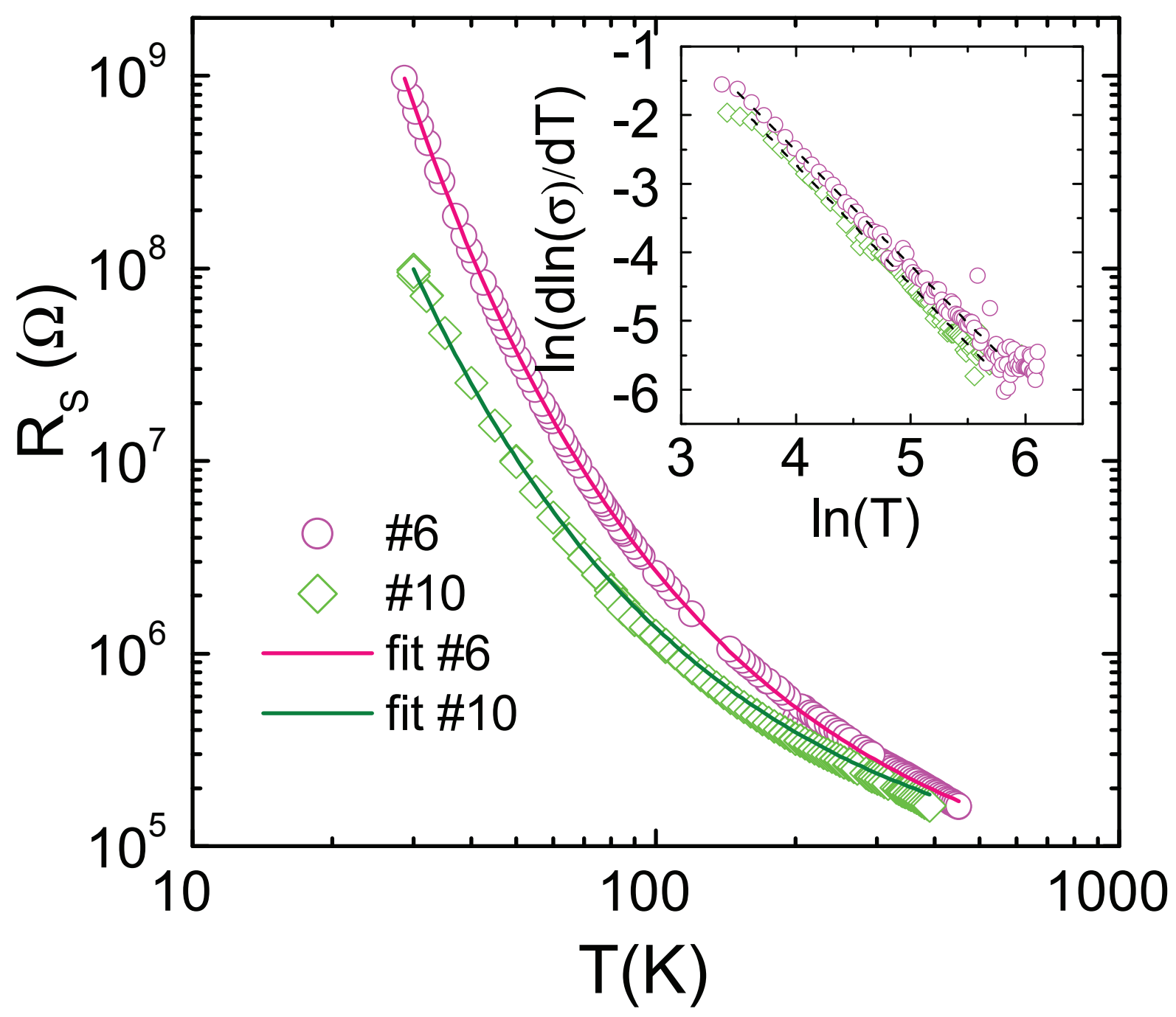

FIG. 10. (a) Sheet resistance $R_{S}$ temperature dependence of the two non metallic samples measured using mesa-etched Hall bars. The inset show the logarithmic fit used to determine the hopping coefficient of 0.7 .

and (c). As the concentration of impurities increases (here boron atoms) the impurity band starts to vanish and join the valence band, forming what it is called a valence band tail ${ }^{38}$. In the case of a heavily doped diamond (above the MIT) the Fermi level lies in the valence band (extended states). In the case of samples \#6 and \#10 where the boron concentration seems to be below the $N_{\text {crit }}$ of the MIT, the Fermi level could be localized in the valence band tail, below the "mobility edge", in an energy range where the mobility is known to be very low compared to that in the valence band ${ }^{39}$. 
In fact, the mobility values of samples \#6 and \#10 are far smaller than the already low $3.6 \mathrm{~cm}^{2} /$ V.s value measured for metallic samples (with Fermi level in the extended sates) which is described in the next section. At 200K, $\mu \sim 0.3 \mathrm{~cm}^{2} / \mathrm{V}$.s. Recently, such low mobility values $\left(0.01 \mathrm{~cm}^{2} / \mathrm{V} . \mathrm{s}\right.$ to $0.1 \mathrm{~cm}^{2} / \mathrm{V}$.s $)$ with comparable sheet carrier densities $(2$ to $4 \times 10^{13} \mathrm{~cm}^{-2}$ ) were reported ${ }^{13}$ for delta layers where the conduction was demonstrated to occur by a variable range hopping mechanism (with a hopping exponent of $1 / 4$ ). In our case a conventional hopping exponent such as $x=1, x=1 / 4$ or $x=1 / 2$ was not found, so that a clear determination of the hopping type (respectively nearest-neighbour, Mott or Efros-Shklovskii variable range hopping) was not possible ${ }^{40}$. An anomalous dependence of $R_{S}$ on temperature with $x=0.7$ (see the inset of figure 10) was found for samples $\# 6$ and \#10. A hopping exponent of $\simeq 0.7$ has already been observed in ultrathin films ${ }^{41-44}$ but its origin was not fully understood. According to Ref.45, it may be a particular case of Efros-Shklovskii variable range hopping in the case of a thin insulating layer with a high dielectric constant embedded between two insulating layers with much lower dielectric constant. In our case, this hopping mechanism with $x=0.7$ was observed in two samples grown in different types of reactors by using two different growth techniques. The fits using Eq. 1 plotted on figure 10 give $T_{0}=780 \mathrm{~K}$ and $T_{0}=540 \mathrm{~K}$ for $\# 6$ and $\# 10$ respectively. Gruenewald et al. ${ }^{46}$ reported a mobility temperature dependence $\mu(T)=\mu_{0} \cdot \exp \left(T_{0} / T\right)^{x}$ in the case of conventional variable range hopping with $x=1 / 4$. In our sample, the same hopping exponent temperature dependance of 0.7 was found for the mobility, but with a $T_{0}$ value different from that of the resistance.

\section{B. METALLIC-LIKE DELTA LAYERS}

Figure 9 (a) shows the temperature dependence of the sheet resistance $R_{S}$ for the different samples. Samples \#3 and \#4 gave a sheet resistance independent of $T$ and a finite conductivity at low temperature, which is typical of a metallic behavior. As shown on figure 9 (b) and (c), the sheet carrier density and the mobility of the six samples (\#2, \#3, \#4, \#5, \#7 and \#8) was constant over the temperature range $50 \mathrm{~K}<T<400 \mathrm{~K}$, which confirmed the metallic behavior. When the doping lies above the critical value inducing MIT $^{35}$, the Fermi level is no longer in the gap but in the valence band, meaning that all dopants are ionized whatever the temperature. The sheet resistance at $300 \mathrm{~K}$ varied from $500 \Omega$ for the 


\begin{tabular}{ccccc}
\hline \hline Samples & $G_{s 0}$ & $R_{s 0}$ & $A$ & $B$ \\
& $(1 / \Omega)$ & $(\mathrm{k} \Omega)$ & $\left(\mathrm{K}^{1 / 2} / \Omega\right)$ & $\left(\mathrm{K}^{1 / 2} / \Omega\right)$ \\
\hline$\# 2$ & $6.3 \times 10^{-6}$ & 158 & $7.6 \times 10^{-6}$ & $2.1 \times 10^{-8}$ \\
$\# 5$ & 15 & $5.0 \times 10^{-6}$ & $3.5 \times 10^{-7}$ \\
$\# 7$ & $6.6 \times 10^{-5}$ & 50 & $2.4 \times 10^{-6}$ & $3.3 \times 10^{-7}$ \\
\hline \hline
\end{tabular}

TABLE III. Fitting parameters of weak localization for samples \#2, \#5 and \#7.

sample with the highest sheet carrier density of about $4 \times 10^{15} \mathrm{~cm}^{-2}$ to $12 \mathrm{k} \Omega$ for those with the lowest sheet carrier density of $1.4 \times 10^{14} \mathrm{~cm}^{-2}$. In all cases the mobility measured was $3.6 \pm 0.8 \mathrm{~cm}^{2} /$ V.s.

\section{Localization}

Samples \#2, \#5, \#7 with higher $R_{S}$ show the same behavior as samples \#3 and \#4 for $\mathrm{T}>70 \mathrm{~K}$. Below this temperature, the sheet resistance shows an upturn which is indicative of partial localization. The conductance $G_{S}=1 / R_{S}$ of these three samples was fitted using a model described in Ref. 35 for bulk material: $G_{S}=G_{S 0}+A T^{1 / 2}+B T$. The term in $T$ takes into account the correction due to weak localization effects while the term in $T^{1 / 2}$ takes into account the correction due to electron-electron interactions. These fits (solid lines) result in finite conductance values at $0 \mathrm{~K}\left(G_{S 0}=1 / R_{S 0}\right)$ for the three samples as can be seen in figure 11 and in table III. The corresponding $R_{S 0}$ values are $158 \mathrm{k} \Omega$ for \#2, $15 \mathrm{k} \Omega$ for \#5 and $50 \mathrm{k} \Omega$ for sample \#7. While this finite conductivity extrapolated to low temperature seems to indicate a metallic character, the corresponding high sheet resistance values lead us to suspect that measurements performed below $4 \mathrm{~K}$ might reveal an insulating behavior.

\section{Inhomogeneity and current paths}

Sample \#8, could be assumed to be metallic as it was grown using the same process than sample \#7. Moreover, sample \#8 has been measured only for $300 \mathrm{~K}<T<350 \mathrm{~K}$ but shows the same properties as sample \#7 over this temperature range. Sample \#7 shows a mobility increase and a sheet carrier density decrease above $300 \mathrm{~K}$. Below room temperature, the 


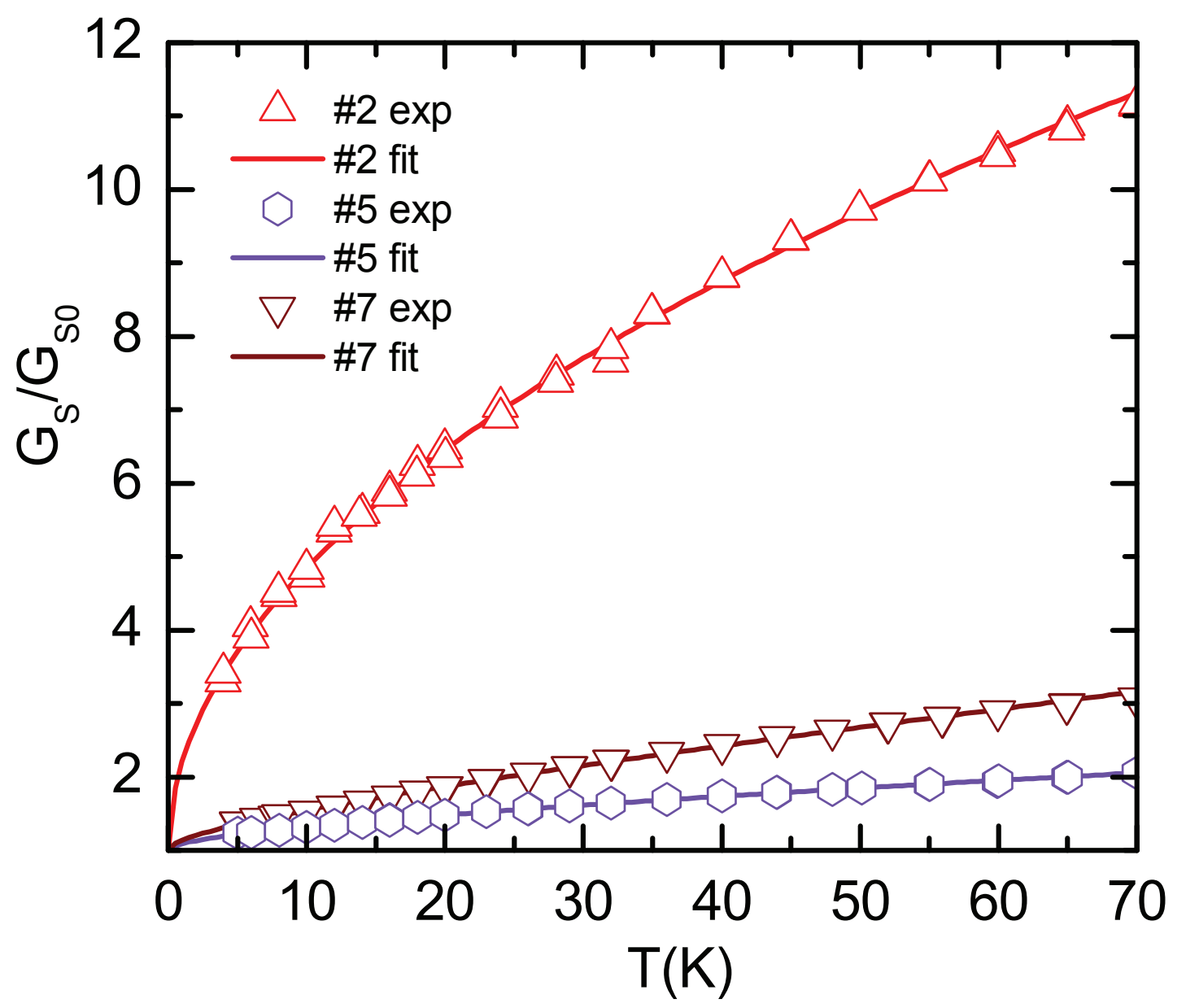

FIG. 11. Temperature dependence of the normalized conductance of samples \#2, \#5 and \#7. The solid lines are the localization fits (see text and Ref. 35)to the experimental data.

observed conduction is typical of a metallic diamond (no $p_{s}$ and $\mu_{H}$ temperature dependence) but for $T>300 \mathrm{~K}$ the mobility starts to increase slowly. This phenomenon could be explained by a parallel conduction path through the buffer and the cap layer (i.e. NiD regions) at high temperatures for which the residual acceptors in the NiD layers start to be thermally ionized and the holes to participate to the conduction as described in Ref. 18. This conduction of the buffer was only observed in this sample even though the buffers of others samples are as thick (or thicker) as that of sample \#7, as can been seen in table IV. Indeed, except for sample \#7, all the samples were grown on Ib-type substrate containing nitrogen at a concentration of a few $10^{19} \mathrm{~cm}^{-3}$. In this case, the main part of the buffer (at least $200 \mathrm{~nm}$ ) is electrically inactive due to the extension of the space charge region 
associated to the $p n$ junction formed by the $p$-type NiD buffer and the $n$-type diamond substrate. The nitrogen content of IIa-type diamond (sample \#7) substrates is much lower, as are the concentrations of other impurities, so that this effect is absent.

Two different Hall bars of the same size were investigated by four probe and Hall effect on sample \#5 (see \#5 HB1 and \#5 HB2 on Fig. 9). The same behaviour and same electrical parameter values were found over the whole temperature range. In addition, the sheet resistance was investigated at $300 \mathrm{~K}$ on Hall bars of different sizes on sample \#7 and \#8 which were expected to involve the same delta structures (same growth conditions) but on two different types of substrates : IIa (100) and Ib (100). As could be expected, no differences between variable Hall bar sizes were observed for the sheet resistance values. However, the distribution of sheet resistance values over the whole sample surface was more homogeneous on sample \#7 $\left(R_{S}\right.$ varying from $11 \mathrm{k} \Omega$ to $\left.12 \mathrm{k} \Omega\right)$ than on sample \#8 $\left(R_{S}\right.$ from $7 \mathrm{k} \Omega$ to $12 \mathrm{k} \Omega$ ). This non homogeneity in sample \#8 is not related to the Hall bar size, but seems to depend on the topography. In fact, as illustrated in Fig. 3, the surface of the substrate used for sample \#8 was far less homogeneous and had more defects and growth sectors than that of sample \#7 (type IIa). Hall effect measurements on sample \#8 show a metallic behavior with a sheet resistance of $12 \mathrm{k} \Omega$ and a mobility of $3.1 \mathrm{~cm}^{2} / \mathrm{V}$.s. As already mentioned, the same value of mobility $\left(\sim 3 \mathrm{~cm}^{2} /\right.$ V.s for $\left.50 \mathrm{~K}<T<450 \mathrm{~K}\right)$ was measured on all samples showing metallic behavior. Consequently, it is highly probable that the Hall bars of sample \#8 where the sheet resistance was measured at value below 12 $\mathrm{k} \Omega$ also have a metallic behavior with the typical $\sim 3 \mathrm{~cm}^{2} / \mathrm{V}$.s mobility value at $300 \mathrm{~K}$. The inhomogeneity is related to the local variation of the sheet carrier density, which should be greater for less resistive Hall bars. Indeed, in degenerate semiconductors, all dopants are ionized and the mobility is dominated by ionized impurity scattering. This is even more the case in diamond, for which the MIT occurs at a high boron concentration ${ }^{36}$. Thus, as the same mobility is measured on all the metallic samples, it can be assumed that this value is related to a constant impurity concentration which is that of the ionized dopant, and these samples have the same doping level. Moreover, all these delta layers were grown using the same process (see table IV) and the only parameters which were varied were the etchback step duration (affecting directly the thickness of the delta layer). Consequently, assuming a constant doping level of the samples, an increase of the sheet resistance corresponds to a 
decrease of the delta layer thickness. The inhomogeneities of $R_{S}$ in sample \#8 are attributed to a thickness inhomogeneity of the delta layer over the sample surface.

\section{Thickness evaluation from electrical properties}

The fabrication of very thin $p^{++}$layers is one of the key issues of boron delta doping. Consequently, the determination of this thickness is a crucial step for such developments. SIMS is commonly used as a tool to obtain depth the profile of dopants and to determine the thickness. However, as discussed in the previous chapter, due to ion mixing and low depth resolution, erroneous values of thickness of epilayers and doping level may be deduced from the raw SIMS data ${ }^{29}$, unless the depth resolution function (DRF) has been determined and is computed with the SIMS data ${ }^{33,34}$. Since this is rarely the case, we chose in this work to investigate only the thick samples by SIMS (except for sample \#TdL2 presented in section III). For the thinnest metallic delta-layers, an evaluation of the maximum thickness from sheet carrier density values was developed, as described below.

In order to evaluate reliably the highly $p$-doped layer thickness, iso-lines corresponding to the measured value of sheet carrier density (iso- $p_{S}$ ) were plotted on a graph showing the boron concentration versus thickness of the $p^{++}$layer (see Fig. 12). For the metallic conduction observed in the samples under study, the boron concentration must be at least equal to $N_{\text {crit }(3 D)}=5 \times 10^{20} \mathrm{~cm}^{-3}$ (critical boron concentration for the MIT ${ }^{35}$ ). So, by plotting the sheet carrier densities $p_{S}$ measured experimentally in metallic samples, the maximum thickness of their delta-doped layers can be directly determined as the intercepts with the horizontal line corresponding to the critical concentration of the MIT transition. For the growth recipes used here, $[\mathrm{B}]>1.5 \times 10^{21} \mathrm{~cm}^{-3}$ was never measured by SIMS, so that a safe higher limit was drawn at $2 \times 10^{21} \mathrm{~cm}^{-3}$. In this manner, the intercept of the lines with $[\mathrm{B}]=$ $2 \times 10^{21} \mathrm{~cm}^{-3}$ gives the delta layer minimum thickness. SIMS data (B-doping and thickness of the delta-layer) are reported on Fig. 12 (a) for samples \#3 and \#4. They are found to be in good agreement with the Hall data $\left(p_{S}=[\mathrm{B}] \times d=10^{21} \times 40 \times 10^{-7}=4 \times 10^{15} \mathrm{~cm}^{-2}\right.$ for sample \#3 blue line and $p_{S}=10^{21} \times 20 \times 10^{-7}=2 \times 10^{15} \mathrm{~cm}^{-2}$ for sample \#4 orange line) confirming the full activation of the boron impurities.

The iso- $p_{S}$ line at $10^{14} \mathrm{~cm}^{-2}$ (lower limit of sheet carrier density at low temperature) of samples \#2, \#7 and \#8 indicates that the thickness of the heavily $p^{++}$layer was equal to 


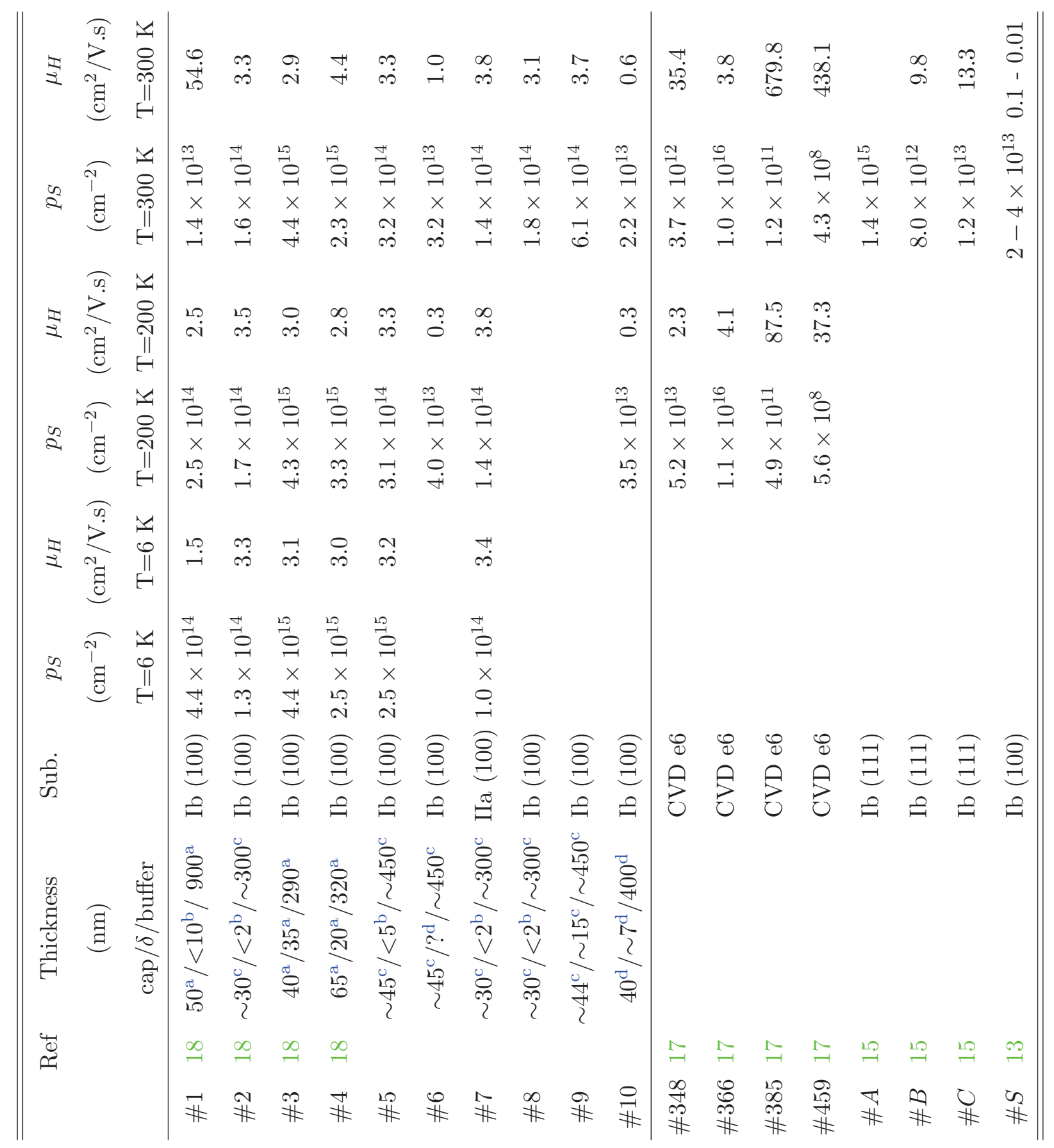

TABLE IV. Summary of thickness of cap layer/delta-layer/buffer layer, substrate type, and electrical transport characteristics of the ten epilayers: Hall mobility and Hall sheet carrier density measured at $6 \mathrm{~K}, 200 \mathrm{~K}$ and $300 \mathrm{~K}$ for samples from this work and for samples from the literature.

${ }^{a}$ Measured by SIMS.

b Measured by Hall effect

${ }^{c}$ Estimated from growth conditions.

d Measured by SIMS for a sample grown under the same conditions. 
or thinner than $2 \mathrm{~nm}$. Thus, we aknowledge that this $2 \mathrm{~nm}$ thickness value was determined under the assumption that the boron ionization energy $E_{a}$ decreased following the same trend as for bulk case. Assuming the bulk properties apply to our case, the deduced thicknesses are low, $<2 \mathrm{~nm}$ for the thinnest, which indicates that this is a good assumption. Indeed, considering $N_{\text {crit }(2 D)}>N_{\text {crit }}$ would lead to lower values of thickness, even lower than one single atomic layer, and so lead to unrealistic values for the sample with the lowest $p_{S}$.
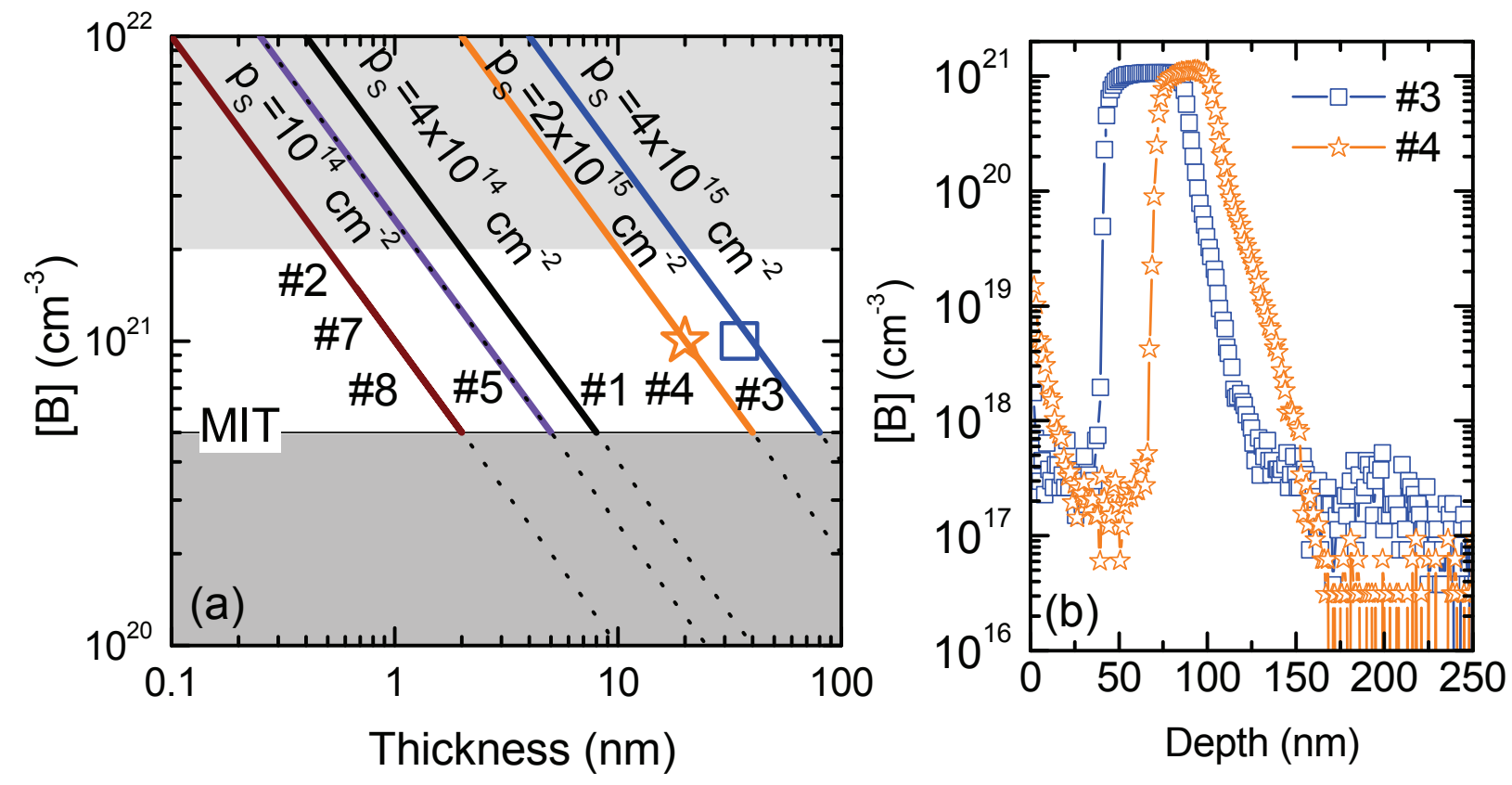

FIG. 12. (a) Sheet carrier density isolines measured by Hall effect, drawn on a boron concentration $\left(\mathrm{cm}^{-3}\right)$ versus thickness $(\mathrm{nm})$ plot. For $[B] \geq 5 \times 10^{20} \mathrm{~cm}^{-3}$ the conduction is metallic. An upper limit was drawn at $2 \times 10^{21} \mathrm{~cm}^{-3}$ (see text). The intercepts of the lines (boundaries between full and dashed) with the horizontal line corresponding to the critical concentration for the MIT yield the maximum thickness of the highly doped region where $[B]>5 \times 10^{20} \mathrm{~cm}^{-3}$, while the intercept of the lines with $[\mathrm{B}]=2 \times 10^{21} \mathrm{~cm}^{-3}$ gives the minimum possible thickness. The symbols are SIMS data for samples \#3 (square) and sample \#4 (star). (b) SIMS boron depth profiles (Cs ${ }^{+}$at 15 $\mathrm{keV}$ ) of the two thickest $p^{++}$layers discussed in this work (\#3 and \#4).

Samples \#2, \#7 and \#8 are the thinnest metallic layers of this work. A structure grown with exactly the same process as samples \#7 and \#8 exhibited a thickness of $1.3 \mathrm{~nm}$ when measured by ellipsometry ${ }^{32}$. Furthermore, the $p^{++}$layers of these two samples were grown under the same conditions as those of the two samples \#TdL1 and \#TdL2 analyzed by TEM 
and SIMS, except for the etching step durations. Delta $\alpha$ (2 nm from STEM-HAADF), delta $\beta(3 \mathrm{~nm})$ and the two delta layers of $\# 7$ and $\# 8$ were etched respectively during 7 min, 6 min and $5 \mathrm{~min}$ in the $\mathrm{H}_{2} / \mathrm{O}_{2}$ mixture, plus 6 min in pure $\mathrm{H}_{2}$. The difference seems to be in good agreement with the difference in thickness determined by SIMS and from electrical properties. For other thin samples $(\# 5, \# 7, \# 8)$, the correlation between etching duration steps and thickness was not obvious. In fact, as nanometric scale values are reached, and with a delta layer thickness of the same order as the roughness of sample surfaces, the control of the thickness and of the doping peak value ${ }^{26}$ becomes a difficult technological challenge. Nevertheless, the delta layer thickness of sample \#5 as deduced from its sheet carrier density ( $5 \mathrm{~nm}$ if doped at $5 \times 10^{20} \mathrm{~cm}^{-3}$, or $2.5 \mathrm{~nm}$ if doped at $1 \times 10^{21} \mathrm{~cm}^{-3}$ as shown on figure 12) was in good agreement with the $3.3 \mathrm{~nm}$ value determined by ellipsometry measurements ${ }^{32}$.

\section{DISCUSSION OF THE MOBILITY}

A mobility value of $3.6 \pm 0.8 \mathrm{~cm}^{2} / \mathrm{Vs}$ was measured in metallic layers (samples \#3, \#4, $\# 5, \# 7, \# 8$ and $\# 9)$ for $6 \mathrm{~K}<T<300 \mathrm{~K}$, whatever the sample sheet carrier densities $\left(\sim 10^{14}\right.$

$\mathrm{cm}^{-3}<p_{s}<\sim 10^{15} \mathrm{~cm}^{-3}$ ), their thicknesses (from less than $2 \mathrm{~nm}$ up to $40 \mathrm{~nm}$ ), or the substrate type they were grown on. Therefore it seems that the scattering mechanisms limiting mobility is not linked to the sheet carrier density as would be the case in two dimensional structures. This is the reason why bulk mobility models are investigated and discussed in view of the experimentally obtained mobility values. For $\mathrm{N}_{A}>5 \times 10^{20} \mathrm{~cm}^{-3}$, the critical concentration for the $\mathrm{MIT}^{35}$, diamond is a degenerate semiconductor. The mobility is dominated by ionized impurities scattering and is independent of temperature. The theoretical mobility of metallic diamond $\left(\mathrm{N}_{A}>5 \times 10^{20} \mathrm{~cm}^{-3}\right)$ was calculated using the method described in Ref. 47 for ionized impurities scattering in the case of a degenerate $p$-type material with negligible phonon scattering ${ }^{36}$. The semiconductor is considered degenerate when $\left(E_{V}-E_{F}\right) / k T \gg 1$ leading to the mobility of ionized impurities for each band:

$$
\mu_{i}=\frac{4 \pi^{2}\left(\epsilon_{r} \epsilon_{0}\right)^{2} \hbar^{3} p_{i}}{N_{I} e^{3} m_{i}^{* 2}\left[\ln \left(1+y_{F}\right)-y_{F} /\left(1+y_{F}\right)\right]}
$$

where $i$ extends over light holes $(l h)$, heavy holes $(h h)$ and holes of the spin-orbit split band (so). $\epsilon_{r}=5.7$ is the diamond dielectric constant, $\epsilon_{0}$ is the vacuum permittivity, $p_{i}$ the number of holes (here equal to the number of acceptors $N_{A}$ as all dopants are ionized in metallic 
diamond), $m_{i}^{*}$ the density of state effective mass of each type of hole, $N_{I}=N_{A}+N_{D}$ is the density of charged ions, $N_{D}$ the compensation, and $y_{F}=3^{1 / 3} 4 \pi^{8 / 3}\left(\epsilon_{r} \epsilon_{r}\right) \hbar^{2} p^{1 / 3} / e^{2} m^{*}$ with $m^{*}$ the total density of state mass ${ }^{36}$. Using the representative effective masses of the three valence sub-bands ${ }^{10}$, the corresponding density of state mass values can be calculated and are $m_{h h}^{*}=0.588 m_{0}, m_{l h}^{*}=0.303 m_{0}$ and $m_{s o}^{*}=0.394 m_{0}$, and the total density of state mass in the absence of additional band splitting $m^{*}=\left(m_{l h}^{* 3 / 2}+m_{h h}^{* 3 / 2}+m_{s o}^{* 3 / 2}\right)^{2 / 3}=$ $0.908 m_{0}$. The mobility of the three valence bands can then be calculated. Assuming that $E_{V}-E_{F}>5 k T$ for a degenerate semiconductor, the product of the density of states with the states occupation probability used to calculate the free carrier density can be written as:

$$
p=\frac{1}{3 \pi^{2}}\left(\frac{2 m^{*}\left(E_{V}-E_{F}\right)}{\hbar^{2}}\right)^{3 / 2}
$$

Introducing the free carrier density into equation 3, we can find the Fermi level position in the valence band. By introducing this value in the same equation and replacing $m^{*}$ by each $m_{i}^{*}$, corresponding value of $p_{i}$ can be found. Knowing the mobility and the density for each hole type and making the approximation that the combined Hall factor $r_{H}$ is equal to one, the theoretical Hall mobility can be calculated :

$$
\mu_{H}=\frac{p_{l h} \mu_{l h}+p_{h h} \mu_{h h}+p_{s o} \mu_{s o}}{p_{l h}+p_{h h}+p_{s o}}
$$

These theoretical mobilities are plotted in Fig. 13 and compared to experimental data from this work and from the literature. Different compensation values were tried to optimally fit the experimental values measured for metallic diamond. As clearly seen in Fig. 13, the measured values of mobility are far below those calculated for low compensation (typically $\left.N_{A}=10^{15} \mathrm{~cm}^{-3}\right)$. Even with larger typical compensation ratios the calculated mobility remains larger than the experimental values. It should be noticed that the value of mobility around $3 \mathrm{~cm}^{2} /$ V.s was not only measured in the samples of this work but also in other published works (see sample \#366 of Ref 17 in table IV) so this mobility value seems to be inherent in heavily doped diamond. We consider a possible solubility of boron in diamond, limiting the boron acceptor density and leading to a boron self-compensation effect. In fact, with very high compensation, the theoretical mobility shrinks toward experimentally measured values.

Even if our calculations cannot fully describe the low mobility value, the dominant, limiting scattering mechanism is most likely associated with ionized impurities. Indeed, if the same 
boron doping level [B] was achieved in the different samples (grown with the same process), then from equation 2 the same $\mu_{i i}$ would be expected for all samples (whatever their thickness) as illustrated in figure 13. This could explain why a similar mobility value is measured in all samples grown using the same recipe.

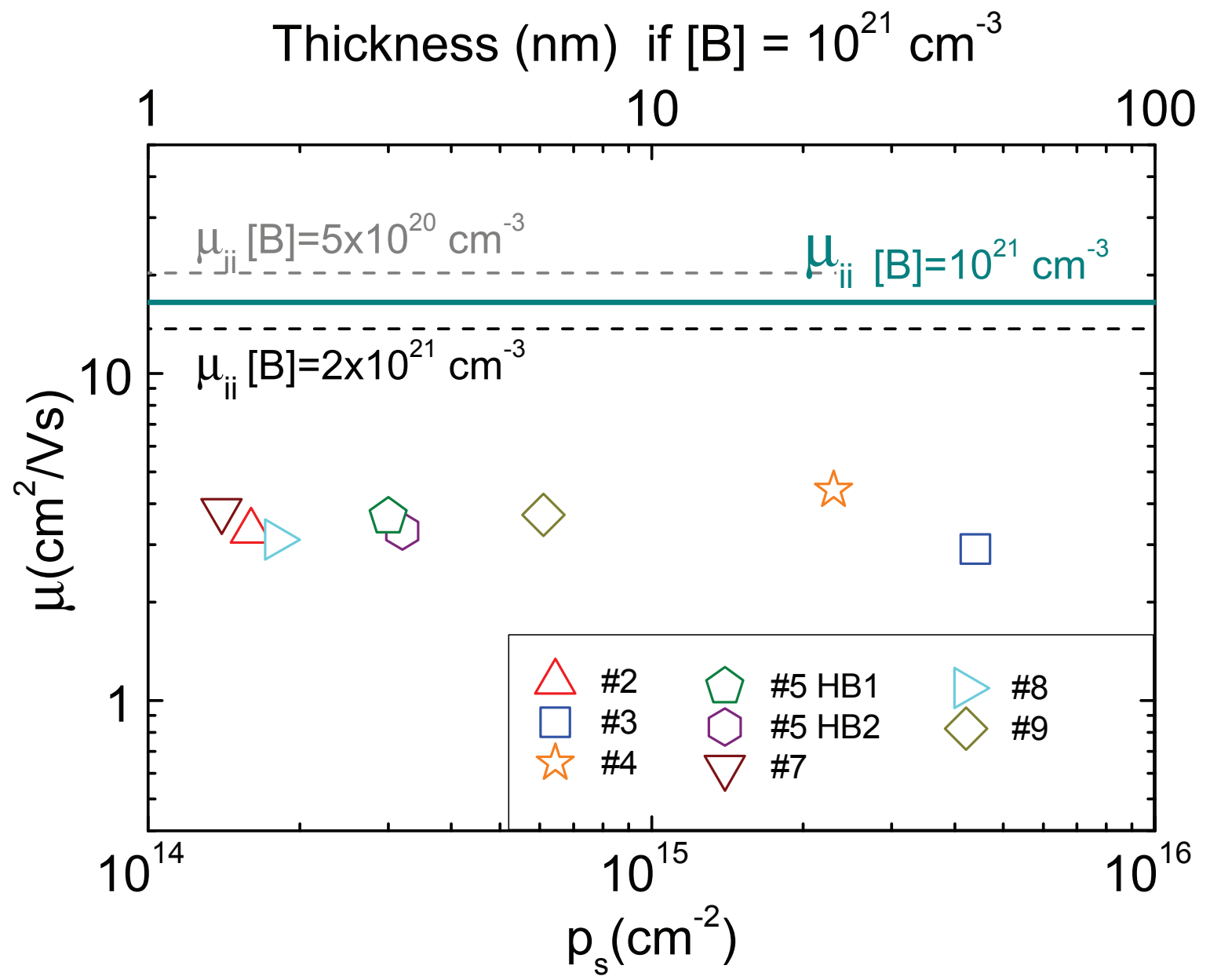

FIG. 13. Experimental mobility versus sheet carrier density of the metallic samples investigated in this work, and calculated mobility values associated to ionized impurity scattering in low compensated bulk diamond with $[\mathrm{B}]$ as indicated.

Nevertheless, the decrease of the layer thickness from $40 \mathrm{~nm}$ down to less than $2 \mathrm{~nm}$ did not improve nor affect the measured mobility value. The thickness of highly B-doped layer in samples \#8 and \#7 is rather low $(<2 \mathrm{~nm})$, but the mobility measured was $3 \mathrm{~cm}^{2} / V . s$ which is typically the same as that measured in a thicker, highly $p$-doped layer like sample \#3 (cf. Fig.9). This result shows that the strong Coulomb scattering induced by ionized 
boron atoms and screened by the free holes (the Fermi screening radius is about $0.3 \mathrm{~nm}$ ) in the delta-layer limits the mobility of the free holes without any sheet density dependence. Since a unique doping process has been used during delta layer growth, we expect a unique maximum boron doping level value (volumic density) in the delta profile (around $1 \times 10^{21}$ $\mathrm{cm}^{-3}$ ). In the case of a bulk scattering mechanism by ionized impurities with the same density of impurities for all samples, such unique mobility value is in agreement with the absence of any sheet density dependence. Indeed, if the sheet density variation is only due to the delta layer thickness variation, the doping level and so the ionized impurities remain constant. No enhancement of the mobility by confinement was observed for a delta layer with $p_{s}=10^{14} \mathrm{~cm}^{-2}$ corresponding to a maximum thickness of $2 \mathrm{~nm}$.

\section{CONCLUSION}

In summary, the present study of the physico-chemical and electronic properties of a series of ten delta-doped diamond structures demonstrated that a nm-scale thickness of the $p^{++}$epilayer can be obtained in a controlled manner using multistep microwave plasma enhanced chemical vapor deposition processes without turning off the plasma. The study confirmed that even in low power reactors, pure hydrogen plasmas etched away the diamond surface at rates which could reach a few $10 \mathrm{~nm} / \mathrm{hr}$. More generally, this work illustrated the difficulty in accurately determining thickness values lower than $5 \mathrm{~nm}$ without retorting to transmission electron microscopy, but that in the case of metallic samples, reliable thickness values in the nm range could be estimated from sheet carrier densities.

For non mettallic layers, the same measurements for samples prepared in two different reactors revealed a hopping conduction mechanism with an anomalous exponent close to 0.7. Further studies must be undertaken to understand the origin of this phenomenon, as well as that of the low mobility value obtained. For metallic delta layers, temperaturedependent Hall effect and four terminal transport measurements confirmed the nanometric thickness measured by other methods, the full activation of boron impurities, the presence of weak localization; and the absence of any mobility enhancement. A constant mobility value $3.6 \pm 0.8 \mathrm{~cm}^{2} / \mathrm{Vs}$ was measured independently of the thickness or substrate type. Within the usual approximations such as that of a parabolic valence band, the bulk mobility which we calculated taking into account quantitatively the scattering by ionized impurities was a 
factor four higher than the experimental mobility value.

\section{ACKNOWLEDGMENTS}

We would like to thank the Agence Nationale de la Recherche (France) for funding under Contract No. ANR08-BLAN-0195, the Région Rhône-Alpes (Project DIAMOND POWER), the Central Electron Microscopy Facilities of the University of Cádiz for technical support, the Ministerio de Ciencia e Innovación (MICINN) of the Spanish Government for funding under Grant No. TEC2009-11399 and EEBB-I-13-07696. We thank Etienne Gheeraert (Institut Néel) for the fruitful discussion and also the technical assistance from the Nanofab team of the Institut Néel, in particular B. Fernandez and T. Crozes.

\section{REFERENCES}

${ }^{1}$ R. S. Balmer, I. Friel, S. Woollard, C. J. Wort, G. . Scarsbrook, S. Coe, H. El-Hajj, A. Kaiser, A. Denisenko, E. Kohn, et J. Isberg, Phil. Trans. R. Soc. A 366, 251 (2008).

${ }^{2}$ J. P. Lagrange, A. Deneuville, and E. Gheeraert, Diamond and Related Materials 7, 1390 (1998).

${ }^{3}$ P.-N. Volpe, J. Pernot, P. Muret, and F. Omnès, Appl. Phys. Lett. 94, 092102 (2009).

${ }^{4}$ J. Pernot, C. Tavares, E. Gheeraert, E. Bustarret, M. Katagiri, and S. Koizumi, Appl. Phys. Lett. 89, 122111 (2006).

${ }^{5}$ T. Kobayashi, T. Ariki, M. Iwabuchi, T. Maki, S. Shikama, S. Suzuki, J. Appl. Phys. 76, 1977 (1994) .

${ }^{6}$ E.F. Schubert, Delta-doping of Semiconductors (Cambridge University Press, 1996).

${ }^{7}$ A. Fiori, J. Pernot, E. Gheeraert, E. Bustarret, Phys. Status Solidi A 207, 2084 (2010).

${ }^{8}$ S. Birner, S. Hackenbuchner, M. Sabathil, G. Zandler, J. A. Majewski, T. Andlauer, T. Zibold, R. Morschl, A. Trellakis, and P. Vogl, Acta Phys. Pol. A 110, 111 (2006).

${ }^{9}$ S. Birner, T. Zibold, T. Andlauer, T. Kubis, M. Sabathil, A. Trellakis, and P. Vogl, IEEE Trans. Electron. Devices 54, 2137 (2007).

${ }^{10}$ M. Willatzen, M. Cardona and N. E. Christensen, Phys. Rev. B 50, 18054 (1994).

${ }^{11}$ H. El-Hajj, A. Denisenko, A. Kaiser, R.S. Balmer, and E. Kohn, Diamond and Related Materials 17, 1259 (2008). 
${ }^{12}$ A. Aleksov, A. Vescan, M. Kunze, P. Gluche, W. Ebert, E. Kohn, A. Bergmeier, and G. Dollinger, Diamond and Related Materials 8, 941 (1999).

${ }^{13}$ J. Scharpf, A. Denisenko, C.I. Pakes, S. Rubanov, A. Bergmaier, G. Dollinger, C. Pietzka, and E. Kohn, Phys. Status Solidi (a) 210, 2028 (2013).

${ }^{14}$ N. Tumilty, J. Welch, H. Ye, R.S. Balmer, C. Wort, R. Lang, and R.B. Jackman, Appl. Phys. Lett. 94, 052107 (2009).

${ }^{15}$ R. Edgington, S. Sato, Y. Ishiyama, R. Morris, R.B. Jackman, and H. Kawarada, J. Appl. Phys. 111, 033710 (2012).

${ }^{16}$ M. Kunze, A. Vescan, G. Dollinger, A. Bergmaier, and E. Kohn, Carbon 37, 787 (1999).

${ }^{17}$ R.S. Balmer, I. Friel, S. Hepplestone, J. Isberg, M.J. Uren, M.L. Markham, N.L. Palmer, J. Pilkington, P. Huggett, S. Majdi, and R. Lang, J. Appl. Phys. 113, 033702 (2013).

${ }^{18}$ G. Chicot, T.N. Tran Thi, A. Fiori, F. Jomard, E. Gheeraert, E. Bustarret, and J. Pernot, Appl. Phys. Lett. 101, 162101 (2012).

${ }^{19}$ C. Mer-Calfati, N. Habka, A. Ben-Younes, M.-A. Pinault, J. Barjon, and P. Bergonzo, Phys. Status Solidi (a) 206, 1955 (2009).

${ }^{20}$ T.N.Tran Thi, B. Fernandez, D. Eon, E. Gheeraert, J. Härtwig, T. Lafford, A. PerratMabilon, C. Peaucelle, P. Olivero, and E. Bustarret, Phys. Status Solidi (a) 208, 2057 (2011).

${ }^{21}$ Mintres BV www.mintres.com .

${ }^{22}$ Syntek Co., LTD. www.syntek.co.jp .

${ }^{23}$ E. Ziegler, J. Hoszowska, T. Bigault, L. Peverini, J.Y. Massonnat, and R. Hustache, AIP Conference Proceedings, 705, 405 (2004).

${ }^{24}$ R.C. Burns, A.I. Chumakov, S.H. Connell, D. Dube, H.P. Godfried, J.O. Hansen, J. Hrtwig, J. Hoszowska, F. Masiello, L. Mkhonza, M. Rebak, A. Rommevaux, R. Setshedi, and P. Van Vaerenbergh, J Phys Condens Matter 21, 364224 (2009).

${ }^{25}$ N. Habka, M.-A. Pinault, C. Mer, F. Jomard, J. Barjon, M. Nesladek, and P. Bergonzo, Phys. Stat. Solidi (a) 205, 2169 (2008).

${ }^{26}$ A. Fiori, T. N. Tran Thi, G. Chicot, F. Jomard, F. Omnès, E. Gheeraert, E. Bustarret, Diamond Relat. Mater. 24, 175 (2012).

${ }^{27}$ P.N. Volpe, N. Tranchant, J.C. Arnault, S. Saada, F. Jomard, and P. Bergonzo, Phys. Status Solidi R. 6, 59 (2012). 
${ }^{28}$ P.N. Volpe, J.-C. Arnault, N. Tranchant, G. Chicot, J. Pernot, F. Jomard, and P. Bergonzo, Diamond and Relat. Mater. 22, 136 (2012).

${ }^{29}$ C. Mer-Calfati, N. Tranchant, P.N. Volpe, F. Jomard, S. Weber, P. Bergonzo, and J.C. Arnault, Materials Letters 115, 283 (2014).

${ }^{30}$ D. Araújo, M.P. Alegre, J.C. Piñero, A. Fiori, E. Bustarret, and F. Jomard, Appl. Phys. Lett. 103, 042104 (2013).

${ }^{31}$ K. Ueda, M. Kasu, T. Makimoto, Appl. Phys. Lett. 90, 122102 (2007).

${ }^{32}$ J. Bousquet, G. Chicot, D. Eon, E. Bustarret, Appl. Phys. Lett. 104, 021905 (2014).

${ }^{33}$ A. Fiori, F. Jomard, T. Teraji, S. Koizumi, J. Isoya, E. Gheeraert, and E. Bustarret, Applied Physics Express 6, 045801 (2013).

${ }^{34}$ A. Fiori, F. Jomard, T. Teraji, G. Chicot, and E. Bustarret, Thin Solid Films, 557, 222 (2013).

${ }^{35}$ T. Klein, P. Achatz, J. Kacmarcik, C. Marcenat, F. Gustafsson, J. Marcus, E. Bustarret, J. Pernot, F. Omnès, B.E. Sernelius, C. Persson, A. Ferreira da Silva, and C. Cytermann, Phys. Rev. B 75, 165313 (2007).

${ }^{36}$ J. Pernot, P.N. Volpe, F. Omnès, P. Muret, V. Mortet, K. Haenen, T. Teraji, Phys. Rev. B 81, 205203 (2010).

${ }^{37}$ M. Werner, R. Locher, W. Kohly, D.S. Holmes, S. Klose, and H.J. Fecht, Diamond and Relat. Mater. 6, 308 (1997).

${ }^{38}$ X. Blase, E. Bustarret, C. Chapelier, T. Klein, and C. Marcenat, Nat. Mater. 8, 375 (2009).

${ }^{39}$ C.M. Soukoulis and E.N. Economou, Waves in Random Media 9, 255 (1999).

${ }^{40}$ K. Tsukioka and H. Okushi, Jpn. J. Appl. Phys. 45, 8571 (2006).

${ }^{41}$ D. van der Putten, J.T. Moonen, H.B. Brom, J.C.M. Brokken-Zijp, and M.A.J. Michels, Phys. Rev. Lett. 69, 494 (1992).

${ }^{42}$ F.W. Van Keuls, X.L. Hu, H.W. Jiang, and A.J. Dahm, Phys. Rev. B 56, 1161 (1997).

${ }^{43}$ C.J. Adkins and E.G. Astrakharchik, J. Phys.: Condens. Matter 10, 6651 (1998).

${ }^{44}$ N. Marković, C. Christiansen, D.E. Grupp, A.M. Mack, G. Martinez-Arizala, and A.M. Goldman, Phys. Rev. B 62, 2195 (2000).

${ }^{45}$ B.I. Shklovskii, Variable Range Hopping in Thin Film with Large Dielectric Constant, arXiv: 0803.3331(2008). 
${ }^{46}$ M. Gruenewald, H. Mueller, P. Thomas, and D. Wuertz, Solid State Communications 38, 1011 (1981).

${ }^{47}$ D.C. Look, Electrical Characterization of GaAs Materials and Devices (Wiley, Chichester; New York, 1989). 